\title{
Tetrathiomolybdate Complexes of Rhodium(I) with Molybdenum-Rhodium Interactions
}

\begin{abstract}
Nikoletta Xamonaki ${ }^{a}$, Anastasios Asimakopoulos ${ }^{a}$, Anastasios Balafas ${ }^{a}$, Marilena Dasenaki ${ }^{a}$, Ioannis Choinopoulos ${ }^{a}$, Silverio $\operatorname{Coco}^{b}$, Emmanuel Simandiras ${ }^{c^{*}}$ and Spyros Koinis ${ }^{a^{*}}$

${ }^{a}$ Faculty of Chemistry, National and Kapodistrian University of Athens, 15771 Athens, Greece,

${ }^{b}$ IU/CINQUIMA, Química Inorgánica, Facultad de Ciencias, Universitad de Valladolid, 47071 Valladolid, Spain,

${ }^{c}$ Theoretical and Physical Chemistry Institute, National Hellenic Research Foundation, 11635 Athens, Greece.
\end{abstract}

Table of contents:

SI.1 List of Rhodium Tetrathiometalato Complexes

SI.2 ESI-MS of TTMR complexes

SI.3 $\left(\mathrm{NEt}_{4}\right)\left[\left(\eta^{4}-\mathrm{COD}\right) \operatorname{Rh}(\mu-\mathrm{S})_{2} \mathrm{MoS}_{2}\right] \mathrm{X}$-Ray crystal structure

SI.4 Optimized structures of complexes 2, 3, 4, 6, 7 and 8 .

SI.5 TDDFT predicted UV-Vis spectra of complexes $3,6,7$ and 8 .

SI.6 QALE electronic parameters for $\mathrm{P}(\mathrm{O}-\mathrm{o}-\mathrm{Tol})_{3}$

SI.7 Statistical Analysis of the QALE Multiple Regressions 


\section{SI.1 List of Rhodium Tetrathiometalato Complexes}

\begin{tabular}{|c|c|c|}
\hline Core & Formula & Ref. \\
\hline $\mathrm{Rh}^{\mathrm{I}} \mathrm{W}^{\mathrm{VI}}$ & $\left(\mathrm{PPh}_{4}\right)\left[(\mathrm{COD}) \mathrm{Rh}(\mu-\mathrm{S})_{2} \mathrm{WS}_{2}\right]$ & [1] \\
\hline \multirow{2}{*}{$\mathrm{Rh}^{\mathrm{I}} \mathrm{Mo}^{\mathrm{VI}} \mathrm{Rh}^{\mathrm{I}}$} & {$\left[(\mathrm{COD}) \mathrm{Rh}(\mu-\mathrm{S})_{2} \mathrm{Mo}(\mu-\mathrm{S})_{2} \mathrm{Rh}(\mathrm{COD})\right]$} & {$[1],[3]$} \\
\hline & {$\left[\left(\mathrm{PPh}_{3}\right)_{2} \mathrm{Rh}(\mu-\mathrm{S})_{2} \mathrm{Mo}(\mu-\mathrm{S})_{2} \mathrm{Rh}\left(\mathrm{PPh}_{3}\right)_{2}\right]$} & {$[2]$} \\
\hline \multirow{7}{*}{$\mathrm{Rh}^{\mathrm{I}} \mathrm{W}^{\mathrm{VI}} \mathrm{Rh}^{\mathrm{I}}$} & {$\left[(\mathrm{COD}) \operatorname{Rh}(\mu-\mathrm{S})_{2} \mathrm{~W}(\mu-\mathrm{S})_{2} \operatorname{Rh}(\mathrm{COD})\right]^{\top}$} & {$[1]-[3]$} \\
\hline & {$\left[(\mathrm{NBD}) \mathrm{Rh}(\mu-\mathrm{S})_{2} \mathrm{~W}(\mu-\mathrm{S})_{2} \mathrm{Rh}(\mathrm{NBD})\right]$} & {$[3]$} \\
\hline & {$\left[\left(\mathrm{PPh}_{3}\right)_{2} \mathrm{Rh}(\mu-\mathrm{S})_{2} \mathrm{~W}(\mu-\mathrm{S})_{2} \mathrm{Rh}\left(\mathrm{PPh}_{3}\right)_{2}\right]$} & {$[1],[2]$} \\
\hline & {$\left[\left(\mathrm{PPh}_{3}\right)(\mathrm{CO}) \mathrm{Rh}(\mu-\mathrm{S})_{2} \mathrm{~W}(\mu-\mathrm{S})_{2} \mathrm{Rh}(\mathrm{CO})\left(\mathrm{PPh}_{3}\right)\right]$} & {$[1],[2]$} \\
\hline & {$\left[\left(\mathrm{PMePh}_{2}\right)_{2} \mathrm{Rh}(\mu-\mathrm{S})_{2} \mathrm{~W}(\mu-\mathrm{S})_{2} \mathrm{Rh}\left(\mathrm{PMePh}_{2}\right)_{2}\right]$} & {$[2]$} \\
\hline & {$\left[(\mathrm{dppe}) \mathrm{Rh}(\mu-\mathrm{S})_{2} \mathrm{~W}(\mu-\mathrm{S})_{2} \mathrm{Rh}(\mathrm{dppe})\right]$} & {$[1],[2]$} \\
\hline & $\left.\left[(t-\mathrm{BuNC})_{2} \mathrm{Rh} \mu-\mathrm{S}\right)_{2} \mathrm{~W}(\mu-\mathrm{S})_{2} \mathrm{Rh}(t-\mathrm{BuNC})_{2}\right]$ & [3] \\
\hline $\mathrm{Rh}^{\mathrm{I}} \mathrm{W}^{\mathrm{VI}} \mathrm{W}^{0}$ & {$\left[(\mathrm{COD}) \operatorname{Rh}(\mu-\mathrm{S})_{2} \mathrm{~W}(\mu-\mathrm{S})_{2} \mathrm{WCl}(\mathrm{dppe})(\mathrm{NN}=\mathrm{CMePh})\right]^{\llbracket}$} & [7] \\
\hline$\left\{\mathrm{Rh}^{\mathrm{I}} \mathrm{W}^{\mathrm{VI}} \mathrm{Rh}^{\mathrm{I}}\right\}_{2}$ & {$\left[\left\{(\mathrm{COD}) \mathrm{Rh}(\mu-\mathrm{S})_{2} \mathrm{~W}(\mu-\mathrm{S})_{2} \mathrm{Rh}\right\}\left(\mu-\mathrm{PPh}_{2} \mathrm{C} \equiv \mathrm{CPPh}_{2}\right)_{2}\right]$} & {$[1]$} \\
\hline
\end{tabular}

ฯ Structurally characterized

Rh(III) tetrathiometalato(VI) complexes

\begin{tabular}{|c|c|c|}
\hline Core & Formula & Ref. \\
\hline \multirow{2}{*}{$\mathrm{Rh}^{\mathrm{III}} \mathrm{W}^{\mathrm{VI}}$} & {$\left[\left(\mathrm{Cp}^{*}\right)\left(\mathrm{P}(\mathrm{OEt})_{3}\right) \mathrm{Rh}(\mu-\mathrm{S})_{2} \mathrm{WS}_{2}\right]^{\pi}$} & [4] \\
\hline & {$\left[\left(\mathrm{Cp}^{*}\right)\left(\mathrm{PMe}_{3}\right) \operatorname{Rh}(\mu-\mathrm{S})_{2} \mathrm{WS}_{2}\right]^{\pi}$} & [5] \\
\hline \multirow{2}{*}{$\mathrm{Rh}^{\mathrm{III}} \mathrm{W}^{\mathrm{VI}} \mathrm{Rh}^{\mathrm{III}}$} & {$\left[(\mathrm{Cp}) \operatorname{CIRh}(\mu-\mathrm{S})_{2} \mathrm{~W}(\mu-\mathrm{S})_{2} \operatorname{RhCl}(\mathrm{Cp})\right]^{\pi}$} & [3] \\
\hline & {$\left[\left(\mathrm{Cp}^{*}\right) \operatorname{CIRh}(\mu-\mathrm{S})_{2} \mathrm{~W}(\mu-\mathrm{S})_{2} \operatorname{RhCl}\left(\mathrm{Cp}^{*}\right)\right]^{\Phi}$} & [6] \\
\hline $\mathrm{Rh}^{\mathrm{III}}\left(\mathrm{W}^{\mathrm{VI}}\right)_{3}$ & {$\left[\mathrm{Rh}\left(\mathrm{WS}_{4}\right)_{3}\right]^{3-}$} & [3] \\
\hline$\left(\mathrm{Rh}^{\mathrm{III}} \mathrm{W}^{\mathrm{VI}}\right) \mathrm{Cu}^{\mathrm{I}}$ & $\left.\left[\left\{\mathrm{Cp}^{*}\right)\left(\mathrm{P}(\mathrm{OEt})_{3}\right) \mathrm{Rh}\left(\mu-\mathrm{WS}_{4}\right)\right\}_{2} \mathrm{Cu}\right] \mathrm{PF}_{6}$ & [8] \\
\hline $\mathrm{Rh}_{2}{ }_{2}^{\mathrm{III}} \mathrm{W}_{2} \mathrm{Cu}_{4}^{\mathrm{I}}$ & {$\left[\left\{(\mathrm{Cp} *)\left(\mathrm{P}(\mathrm{OEt})_{3}\right) \operatorname{Rh}\left(\mu-\mathrm{WS}_{4}\right)(\mathrm{CuCl}) \mathrm{Cu}\right\}_{2}(\mu-\mathrm{Cl})_{2}\right]^{\pi}$} & [4] \\
\hline
\end{tabular}

${ }^{\top}$ Structurally characterized

[1] Howard, K. E.; Rauchfuss, T, B.; Rheingold, A. L. J. Am. Chem. Soc. 1986, 108, 297-299.

[2] Siedle, A. R.; Gleason, W. B. Inorg. Chem. 1986, 25, 4054-4057.

[3] Howard, K. E.; Rauchfuss, T, B.; Wilson, R.S. Inorg. Chem. 1988, 27, 3561-3567.

[4] Ogo, S.; Suzuki, T.; Ozawa, Y.; Isobe, K. Chem. Lett. 1994, 1235; Inorg. Chem. 1996, 35, 60936101 .

[5] Herberhold, M.; Jin, G.-X., Rheingold, A. L. Z. Anorg. Allg. Chem., 2005, 631, 135-140.

[6] Wanner, M.; Hartenbach, I.; Fiedler J.; Schleid, Th.; Kaim W. Z. Naturforsch. 2001, 56b, 940-946.

[7] Seino, H.; Mizobe, Y.; Hidai M. Bull. Chem. Soc. Jpn. 2000, 73, 631-639.

[8] Ogo, S.; Suzuki, T.; Nomura, S; Asakura, K.; Isobe, K. J. Clust. Sci. 1995, 6, 421-436. 


\section{SI.2 ESI-MS of TTMR complexes}

* $\left[(\mathrm{COD}) \mathrm{Rh}\left(\mathrm{MoS}_{4}\right)\right]^{-} 2$

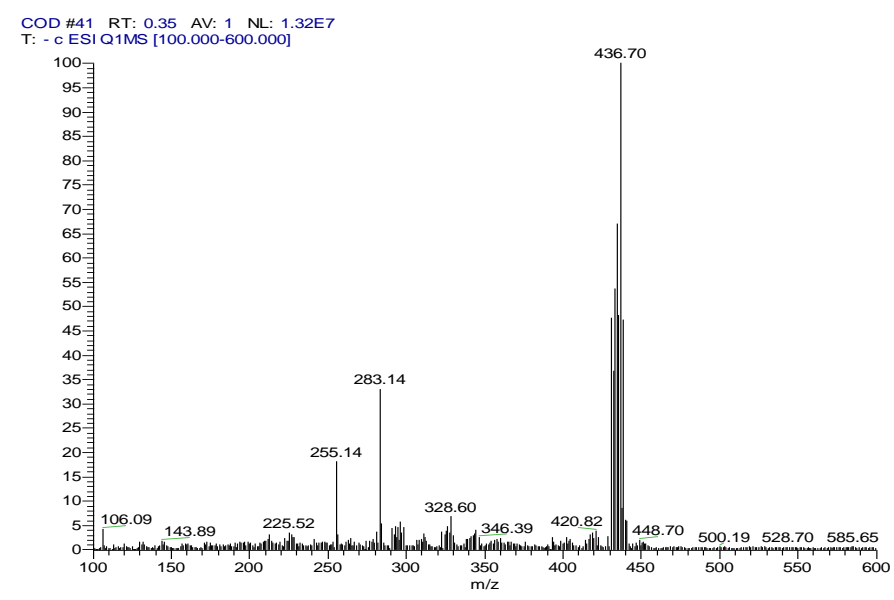

* $\left[\left(\mathrm{P}(\mathrm{OPh})_{3}\right)_{2} \mathrm{Rh}\left(\mathrm{MoS}_{4}\right)\right]^{-} 4$

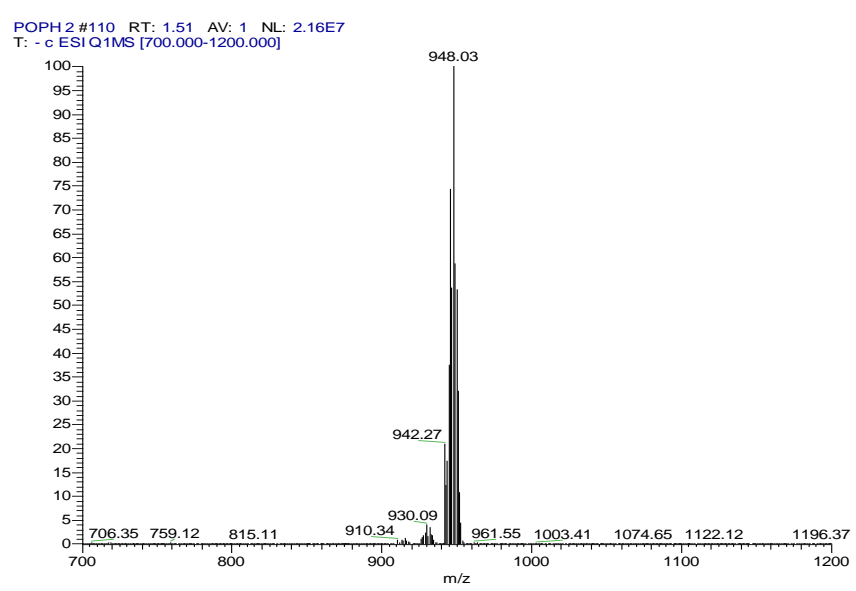


* $\left[\left(\mathrm{P}(\mathrm{O}-o-\mathrm{Tol})_{3}\right)_{2} \mathrm{Rh}\left(\mathrm{MoS}_{4}\right)\right]^{-} 5$

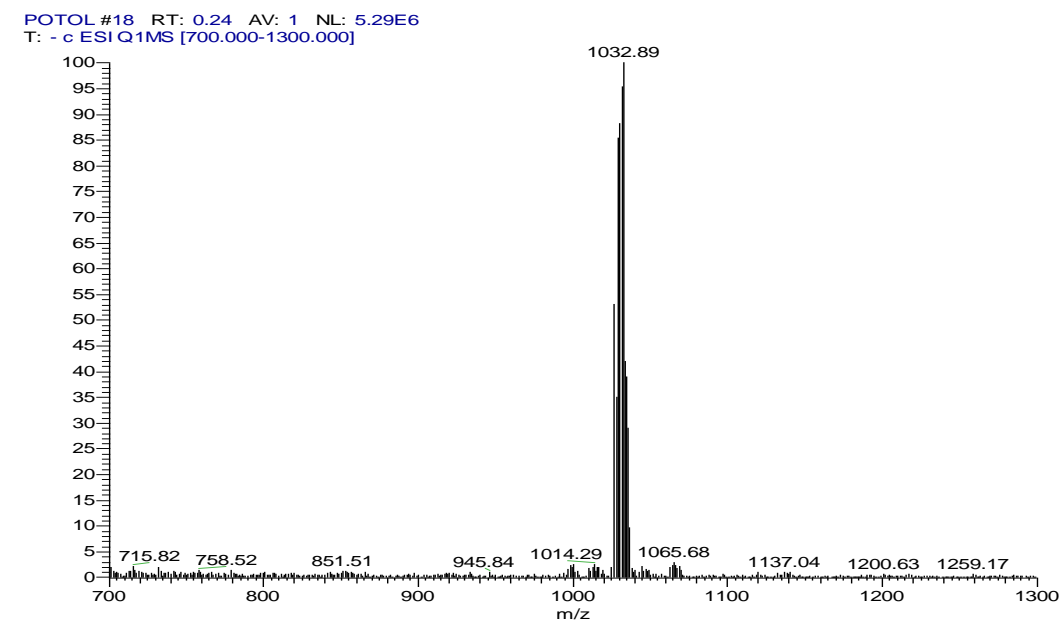

* $\left[\left(\mathrm{P}(\mathrm{OMe})_{3}\right)_{2} \mathrm{Rh}\left(\mathrm{MoS}_{4}\right)\right]^{-} \mathbf{6}$

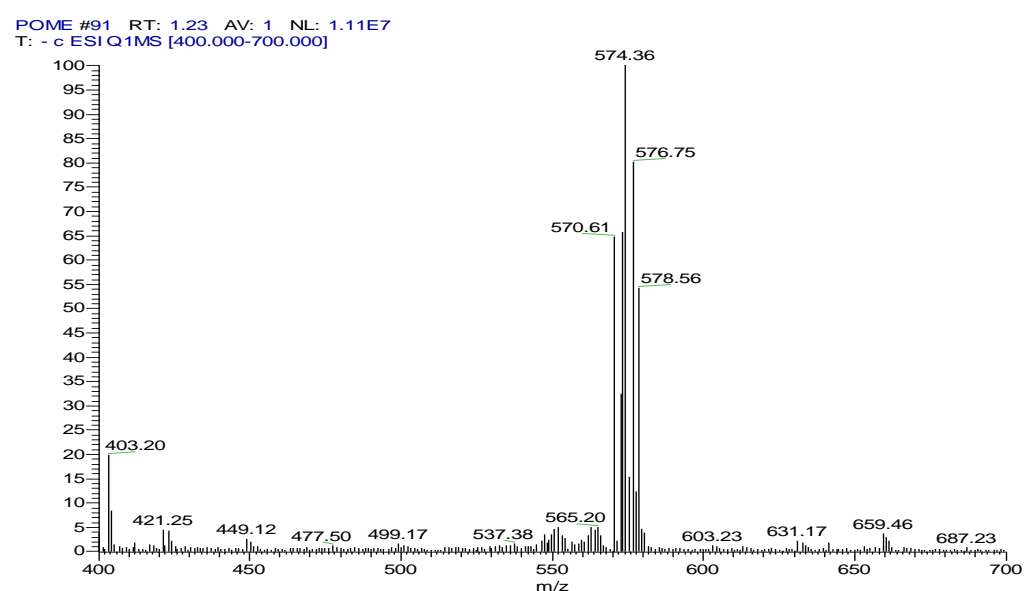


* $\left[\left(\mathrm{P}(\mathrm{OEt})_{3}\right)_{2} \mathrm{Rh}\left(\mathrm{MoS}_{4}\right)\right]^{-} 7$

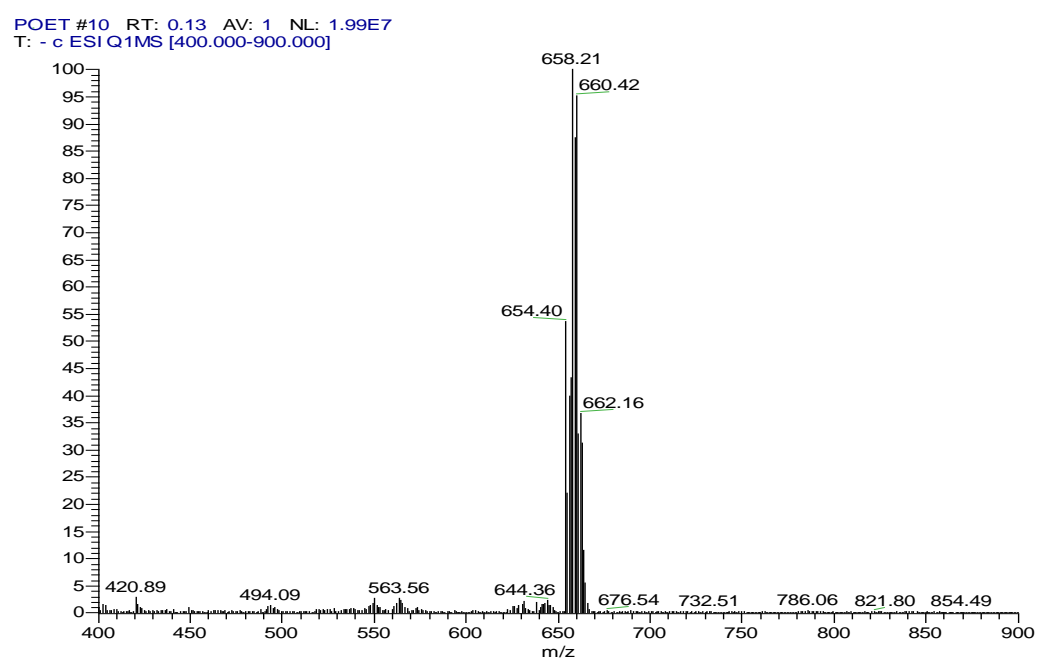

* $\left[\left(\mathrm{P}(\mathrm{O}-i-\mathrm{Pr})_{3}\right)_{2} \mathrm{Rh}\left(\mathrm{MoS}_{4}\right)\right]^{-} 8$

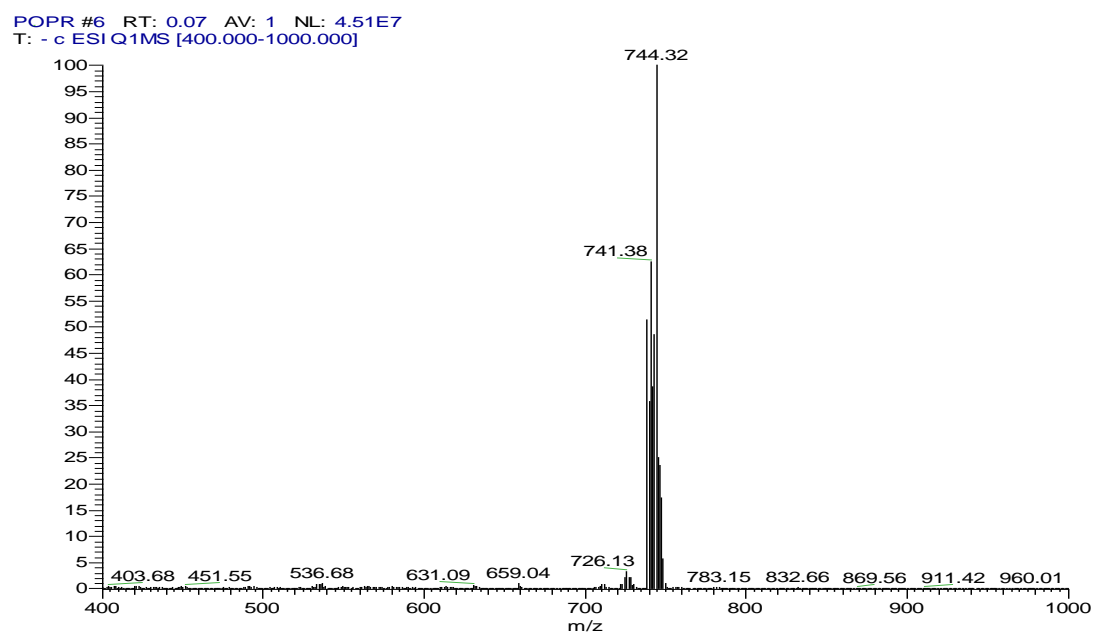


* $\left[\left(\mathrm{P}(\text { cis-dppen }) \mathrm{Rh}\left(\mathrm{MoS}_{4}\right)\right]^{-} 9\right.$

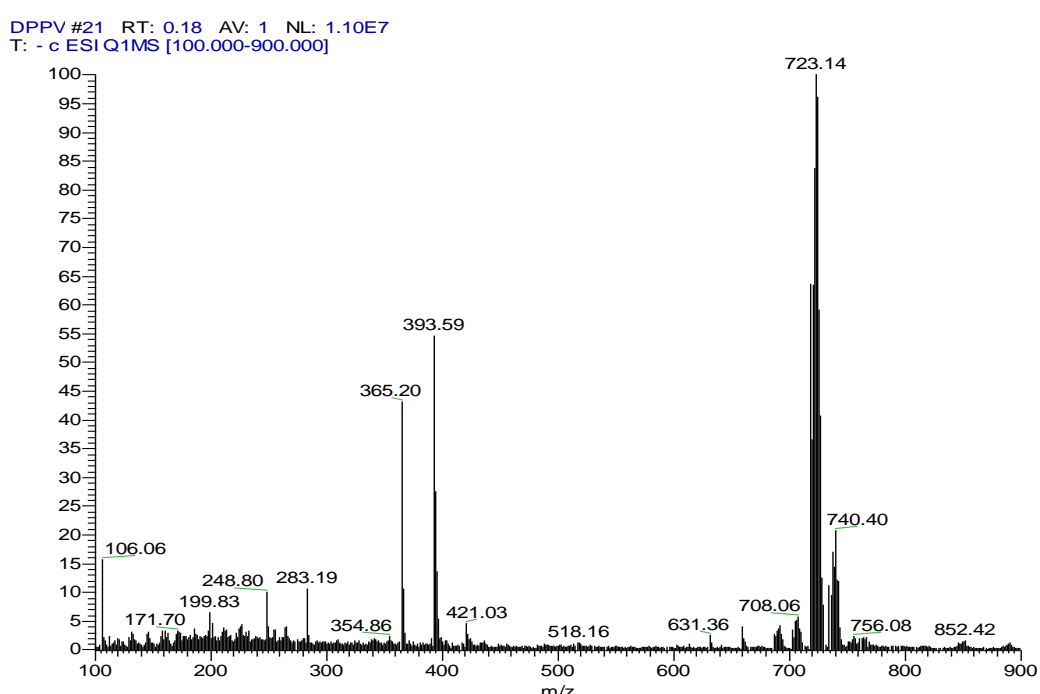

* $\left[\left(\mathrm{P}(\mathrm{dppe}) \mathrm{Rh}\left(\mathrm{MoS}_{4}\right)\right]^{-} \mathbf{1 0}\right.$

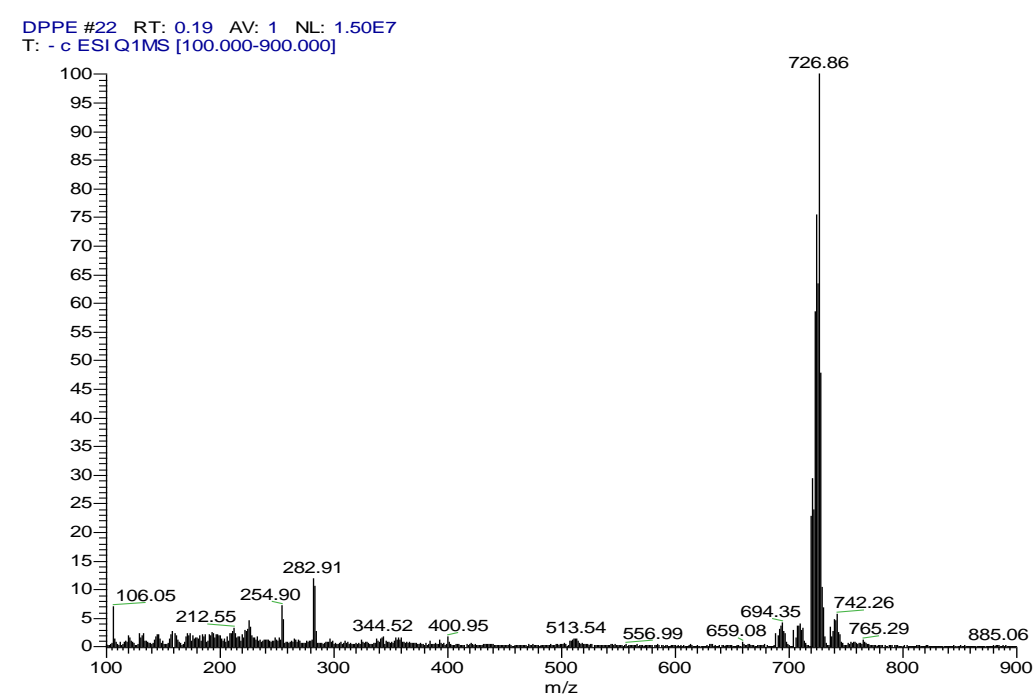


* $\left[\left(\mathrm{P}(\mathrm{dppb}) \mathrm{Rh}\left(\mathrm{MoS}_{4}\right)\right]^{-} \mathbf{1 1}\right.$

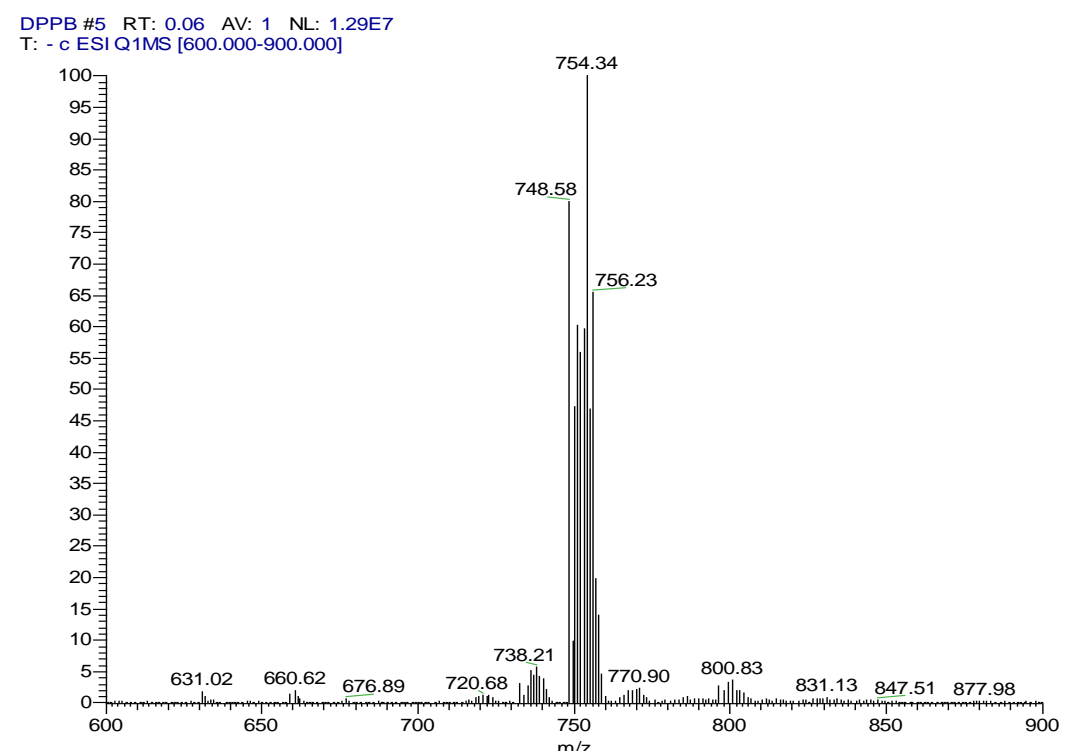




\section{SI.3 $\left(\mathrm{NEt}_{4}\right)\left[\left(\eta^{4}-\mathrm{COD}\right) \operatorname{Rh}(\mu-\mathrm{S})_{2} \mathrm{MoS}_{2}\right] \mathrm{X}$-Ray crystal structure}

Table SI.3.1 Crystal data and structure refinement

\begin{tabular}{|c|c|}
\hline & 2 \\
\hline formula & $\mathrm{C}_{16} \mathrm{H}_{32} \mathrm{MoNRhS}_{4}$ \\
\hline fw & 565.51 \\
\hline$T(\mathrm{~K})$ & $301(2)$ \\
\hline$\lambda(\AA)$ & 0.71073 \\
\hline cryst. syst. & Monoclinic \\
\hline space group & $\mathrm{P} 2{ }_{1} / c$ \\
\hline$a(\AA)$ & $8.588(6)$ \\
\hline$b(\AA)$ & $16.089(11)$ \\
\hline c $(\AA)$ & $16.075(11)$ \\
\hline$\alpha(\operatorname{deg})$ & 90 \\
\hline$\beta(\operatorname{deg})$ & $100.054(11)$ \\
\hline$\gamma(\operatorname{deg})$ & 90 \\
\hline$V\left(\AA^{3}\right)$ & $2187(2)$ \\
\hline $\mathrm{Z}$ & 4 \\
\hline calcd. density $\left(\mathrm{g} / \mathrm{cm}^{3}\right)$ & 1.717 \\
\hline absorption coeff. $\left(\mathrm{mm}^{-1}\right)$ & 1.708 \\
\hline $\mathrm{F}(000)$ & 1144.0 \\
\hline Crystal size $/ \mathrm{mm}^{3}$ & $0.2 \times 0.07 \times 0.06$ \\
\hline Radiation & $\operatorname{MoK} \alpha(\lambda=0.71073)$ \\
\hline $2 \Theta$ range for data collection $/^{\circ}$ & 3.61 to 56.666 \\
\hline Index ranges & $-11 \leq \mathrm{h} \leq 11,-21 \leq \mathrm{k} \leq 21,-21 \leq 1 \leq 21$ \\
\hline Reflections collected & 20487 \\
\hline Independent reflections & $5435\left[\mathrm{R}_{\mathrm{int}}=0.0375, \mathrm{R}_{\text {sigma }}=0.0341\right]$ \\
\hline Refinement method & Full-matrix least-squares on F2 \\
\hline data / restraints / parameters & $5435 / 0 / 213$ \\
\hline Goodness-of-fit on F2 & 1.077 \\
\hline Final $R$ indexes $[\mathrm{I}>=2 \sigma(\mathrm{I})]$ & $\mathrm{R}_{1}=0.0304, \mathrm{wR}_{2}=0.0932$ \\
\hline Final $\mathrm{R}$ indexes [all data] & $\mathrm{R}_{1}=0.0407, \mathrm{wR}_{2}=0.1011$ \\
\hline largest diff. peak / hole $\left(\mathrm{e} \AA^{-3}\right)$ & 0.65 and -0.39 \\
\hline
\end{tabular}


SI.4. Optimized structures of complexes 2, 3, 4, 6, 7 and 8 . $\left[(\mathrm{COD}) \mathrm{Rh}\left(\mathrm{MoS}_{4}\right)\right]^{-} \mathbf{2}$

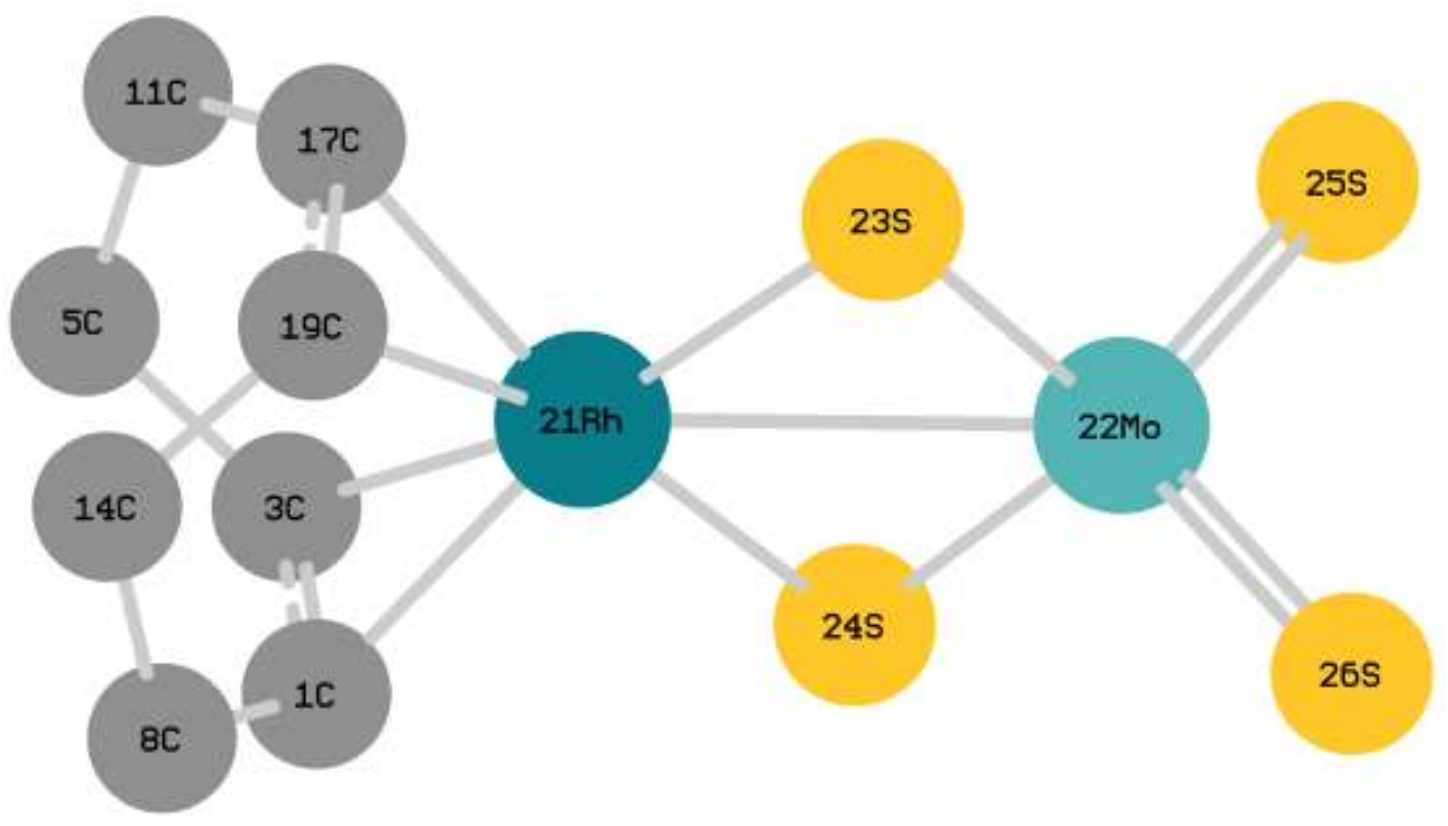

\begin{tabular}{|l|l|}
\hline \multicolumn{2}{|l|}{ Bond Lengths $(\stackrel{\AA}{)})$} \\
\hline $21 \mathrm{Rh}-1 \mathrm{C}$ & 2.163 \\
\hline $21 \mathrm{Rh}-3 \mathrm{C}$ & 2.191 \\
\hline $21 \mathrm{Rh}-17 \mathrm{C}$ & 2.163 \\
\hline $21 \mathrm{Rh}-19 \mathrm{C}$ & 2.191 \\
\hline $21 \mathrm{Rh}-23 \mathrm{~S}$ & 2.372 \\
\hline $21 \mathrm{Rh}-24 \mathrm{~S}$ & 2.372 \\
\hline $22 \mathrm{Mo}-23 \mathrm{~S}$ & 2.253 \\
\hline $22 \mathrm{Mo}-24 \mathrm{~S}$ & 2.253 \\
\hline $21 \mathrm{Rh}-22 \mathrm{Mo}$ & 2.990 \\
\hline $22 \mathrm{Mo}-25 \mathrm{~S}$ & 2.156 \\
\hline $22 \mathrm{Mo}-26 \mathrm{~S}$ & 2.158 \\
\hline
\end{tabular}

\begin{tabular}{|l|l|}
\hline \multicolumn{2}{|l|}{ Bond Angles (deg) } \\
\hline 1C-21Rh-3C & 37.50 \\
\hline 17C-21Rh-19C & 37.50 \\
\hline 21Rh-23S-22Mo & 80.51 \\
\hline 23S-22Mo-24S & 102.97 \\
\hline 22Mo-24S-21Rh & 80.51 \\
\hline 24S-21Rh-23S & 96.01 \\
\hline 25S-22Mo-26S & 109.93 \\
\hline
\end{tabular}




\section{$\left[(\mathrm{CO})_{2} \mathrm{Rh}\left(\mathrm{MoS}_{4}\right)\right]^{-} \mathbf{3}$}

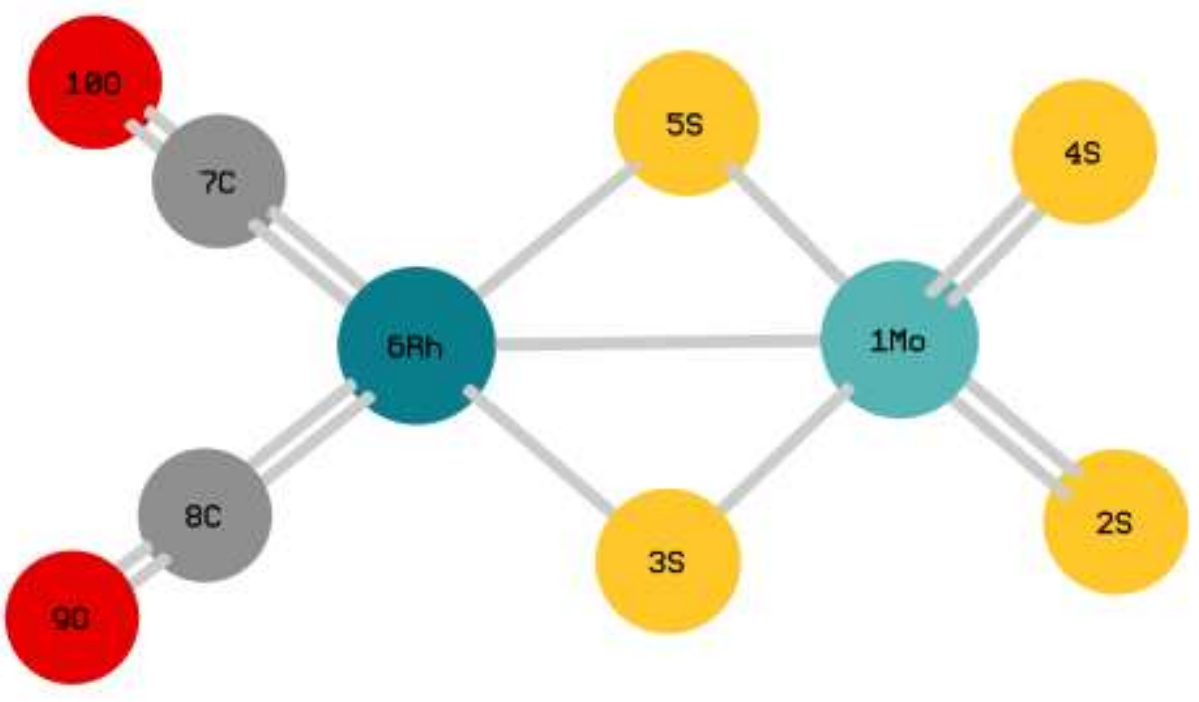

\begin{tabular}{|c|c|}
\hline \multicolumn{2}{|c|}{ Bond Lengths $(\AA)$} \\
\hline $7 \mathrm{C}-10 \mathrm{O}$ & 1.145 \\
\hline $8 \mathrm{C}-9 \mathrm{O}$ & 1.145 \\
\hline 6Rh-7C & 1.864 \\
\hline 6Rh-8C & 1.864 \\
\hline 6Rh-3S & 2.411 \\
\hline 6Rh-5S & 2.411 \\
\hline $1 \mathrm{Mo}-3 \mathrm{~S}$ & 2.258 \\
\hline $1 \mathrm{Mo}-5 \mathrm{~S}$ & 2.258 \\
\hline $6 \mathrm{Rh}-1 \mathrm{Mo}$ & 2.994 \\
\hline $1 \mathrm{Mo}-2 \mathrm{~S}$ & 2.151 \\
\hline $1 \mathrm{Mo}-4 \mathrm{~S}$ & 2.151 \\
\hline
\end{tabular}

\begin{tabular}{|l|l|}
\hline \multicolumn{2}{|l|}{ Bond Angles $(\mathbf{d e g})$} \\
\hline 7C-6Rh-8C & 94.19 \\
\hline 6Rh-5S-1Mo & 79.70 \\
\hline 5S-1Mo-3S & 104.78 \\
\hline 1Mo-3S-6Rh & 79.70 \\
\hline 3S-6Rh-5S & 95.82 \\
\hline 2S-1Mo-4S & 109.42 \\
\hline
\end{tabular}


$\left[\left(\mathrm{P}(\mathrm{OPh})_{3}\right)_{2} \mathrm{Rh}\left(\mathrm{MoS}_{4}\right)\right]^{-} \quad 4$

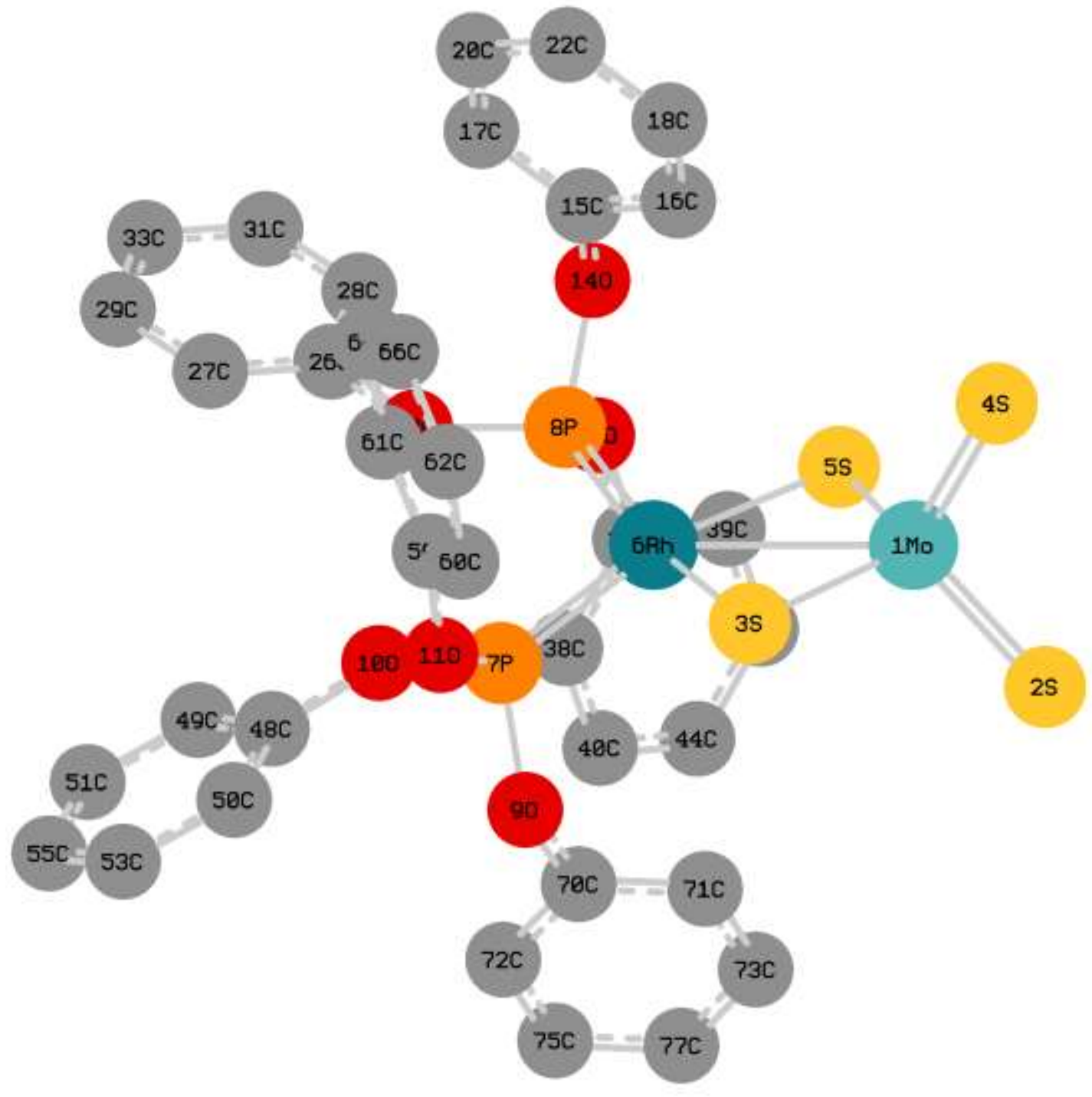

\begin{tabular}{|l|l|}
\hline \multicolumn{2}{|l|}{ Bond Lengths $(\stackrel{\AA}{)}$} \\
\hline $7 \mathrm{P}-6 \mathrm{Rh}$ & 2.206 \\
\hline $8 \mathrm{P}-6 \mathrm{Rh}$ & 2.206 \\
\hline $6 \mathrm{Rh}-3 \mathrm{~S}$ & 2.409 \\
\hline $6 \mathrm{Rh}-5 \mathrm{~S}$ & 2.409 \\
\hline $1 \mathrm{Mo}-3 \mathrm{~S}$ & 2.252 \\
\hline $1 \mathrm{Mo}-5 \mathrm{~S}$ & 2.252 \\
\hline $6 \mathrm{Rh}-1 \mathrm{Mo}$ & 2.987 \\
\hline $1 \mathrm{Mo}-2 \mathrm{~S}$ & 2.156 \\
\hline $1 \mathrm{Mo}-4 \mathrm{~S}$ & 2.156 \\
\hline
\end{tabular}

\begin{tabular}{|l|l|}
\hline \multicolumn{2}{|l|}{ Bond Angles (deg) } \\
\hline 7P-6Rh-8P & 101.83 \\
\hline 6Rh-5S-1Mo & 79.63 \\
\hline 5S-1Mo-3S & 105.02 \\
\hline 1Mo-3S-6Rh & 79.63 \\
\hline 3S-6Rh-5S & 95.72 \\
\hline 2S-1Mo-4S & 110.15 \\
\hline
\end{tabular}


$\left[\left(\mathrm{P}(\mathrm{OMe})_{3}\right)_{2} \mathrm{Rh}\left(\mathrm{MoS}_{4}\right)\right]^{-} \quad \mathbf{6}$

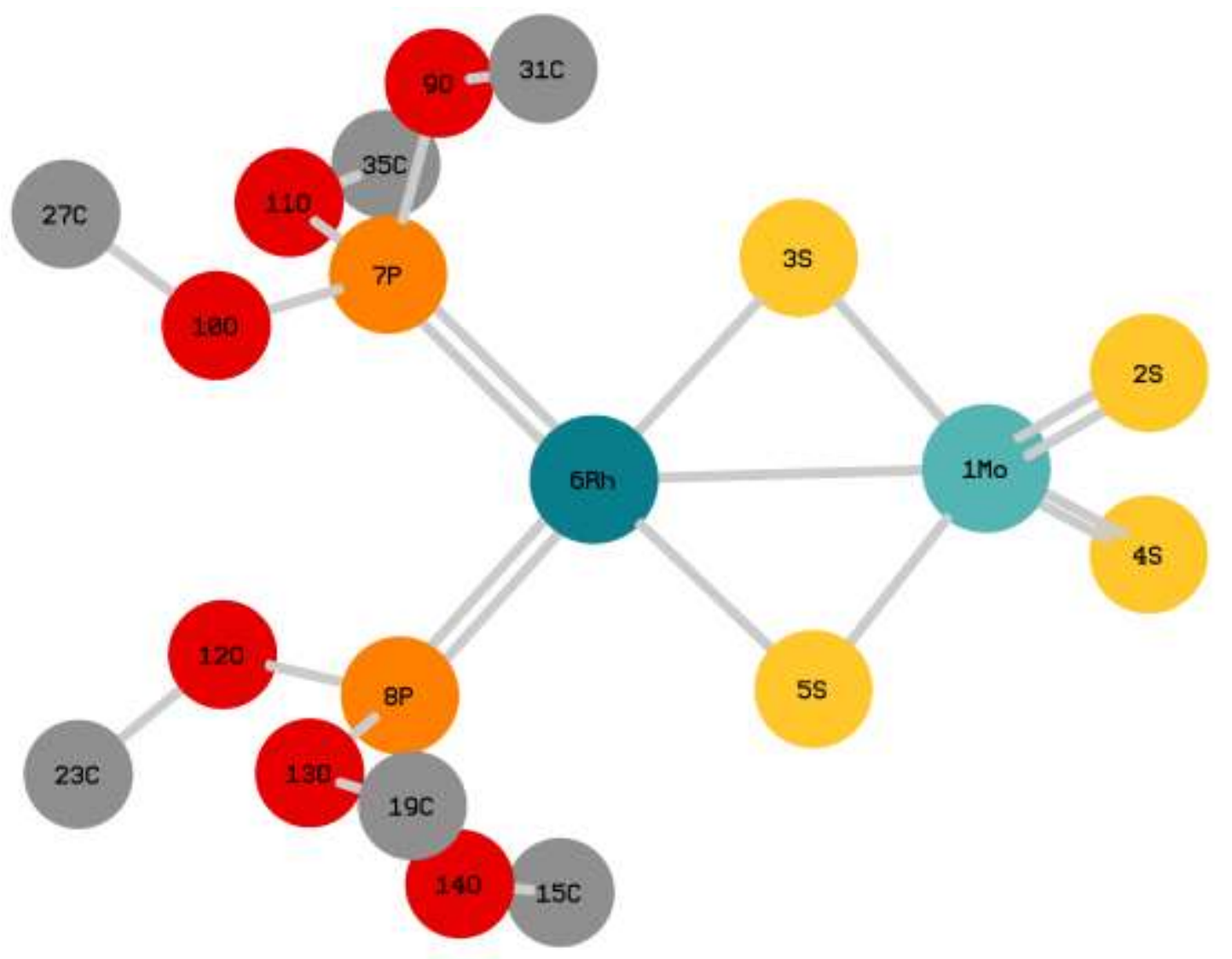

\begin{tabular}{|l|l|}
\hline \multicolumn{2}{|l|}{ Bond Lengths $(\stackrel{\AA}{)}$} \\
\hline 7P-6Rh & 2.207 \\
\hline $8 \mathrm{P}-6 \mathrm{Rh}$ & 2.207 \\
\hline $6 \mathrm{Rh}-3 \mathrm{~S}$ & 2.409 \\
\hline $6 \mathrm{Rh}-5 \mathrm{~S}$ & 2.409 \\
\hline $1 \mathrm{Mo}-3 \mathrm{~S}$ & 2.253 \\
\hline $1 \mathrm{Mo}-5 \mathrm{~S}$ & 2.253 \\
\hline $6 \mathrm{Rh}-1 \mathrm{Mo}$ & 2.983 \\
\hline $1 \mathrm{Mo}-2 \mathrm{~S}$ & 2.161 \\
\hline $1 \mathrm{Mo}-4 \mathrm{~S}$ & 2.161 \\
\hline
\end{tabular}

\begin{tabular}{|l|l|}
\hline \multicolumn{2}{|l|}{ Bond Angles (deg) } \\
\hline 7P-6Rh-8P & 92.97 \\
\hline 6Rh-5S-1Mo & 79.52 \\
\hline 5S-1Mo-3S & 105.09 \\
\hline 1Mo-3S-6Rh & 79.63 \\
\hline 3S-6Rh-5S & 95.87 \\
\hline 2S-1Mo-4S & 110.12 \\
\hline
\end{tabular}




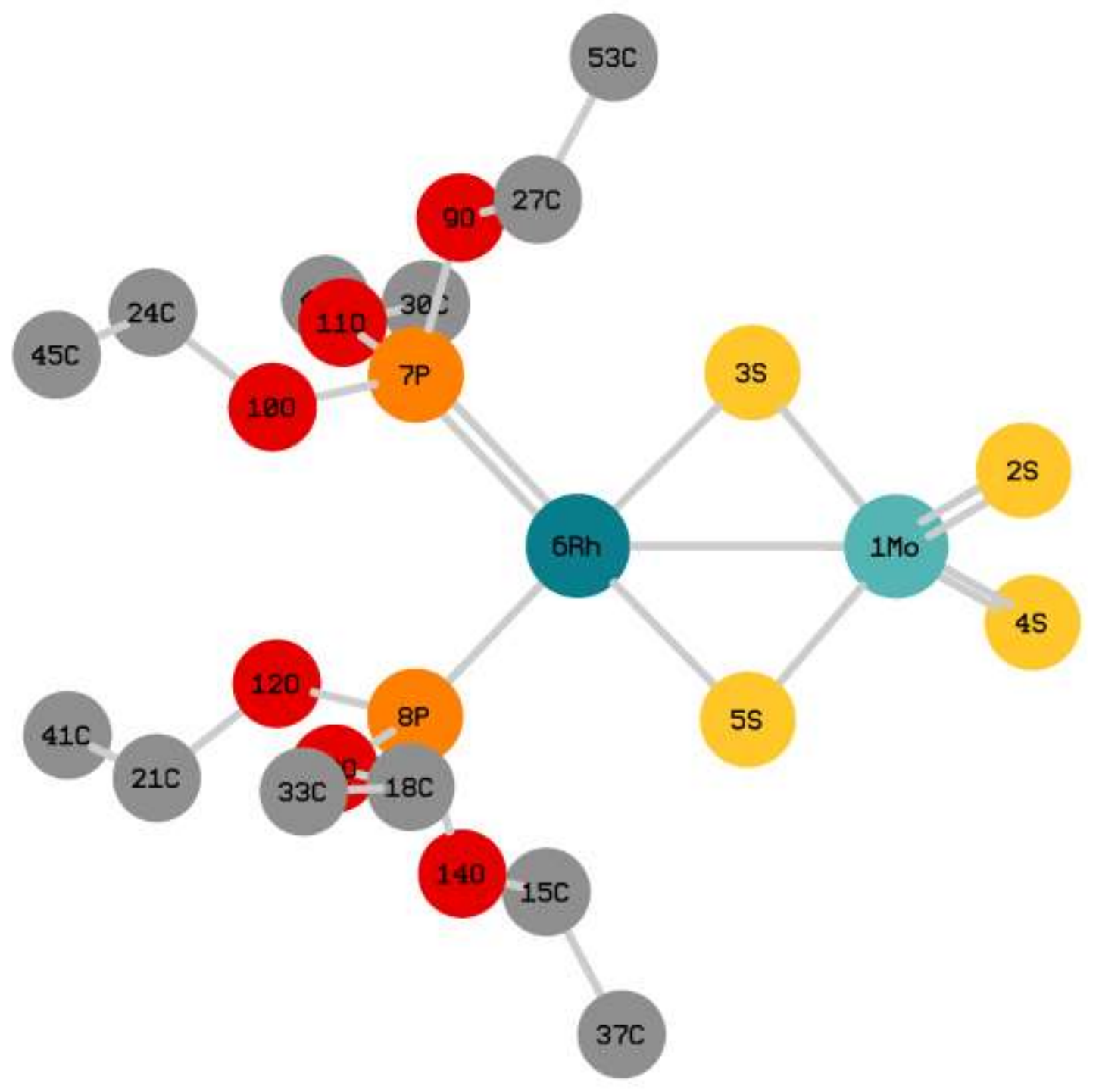

\begin{tabular}{|l|l|}
\hline \multicolumn{2}{|l|}{ Bond Lengths $(\mathbf{\AA})$} \\
\hline 7P-6Rh & 2.212 \\
\hline 8P-6Rh & 2.212 \\
\hline $6 \mathrm{Rh}-3 \mathrm{~S}$ & 2.409 \\
\hline $6 \mathrm{Rh}-5 \mathrm{~S}$ & 2.409 \\
\hline $1 \mathrm{Mo}-3 \mathrm{~S}$ & 2.252 \\
\hline $1 \mathrm{Mo}-5 \mathrm{~S}$ & 2.252 \\
\hline $6 \mathrm{Rh}-1 \mathrm{Mo}$ & 2.988 \\
\hline $1 \mathrm{Mo}-2 \mathrm{~S}$ & 2.161 \\
\hline $1 \mathrm{Mo}-4 \mathrm{~S}$ & 2.161 \\
\hline
\end{tabular}

\begin{tabular}{|l|l|}
\hline \multicolumn{2}{|l|}{ Bond Angles (deg) } \\
\hline 7P-6Rh-8P & 92.88 \\
\hline 6Rh-5S-1Mo & 79.66 \\
\hline 5S-1Mo-3S & 104.97 \\
\hline 1Mo-3S-6Rh & 79.66 \\
\hline 3S-6Rh-5S & 95.70 \\
\hline 2S-1Mo-4S & 110.05 \\
\hline
\end{tabular}


$\left[\left(\mathrm{P}(\mathrm{O}-i-\mathrm{Pr})_{3}\right)_{2} \mathrm{Rh}\left(\mathrm{MoS}_{4}\right)\right]^{-} \mathbf{8}$

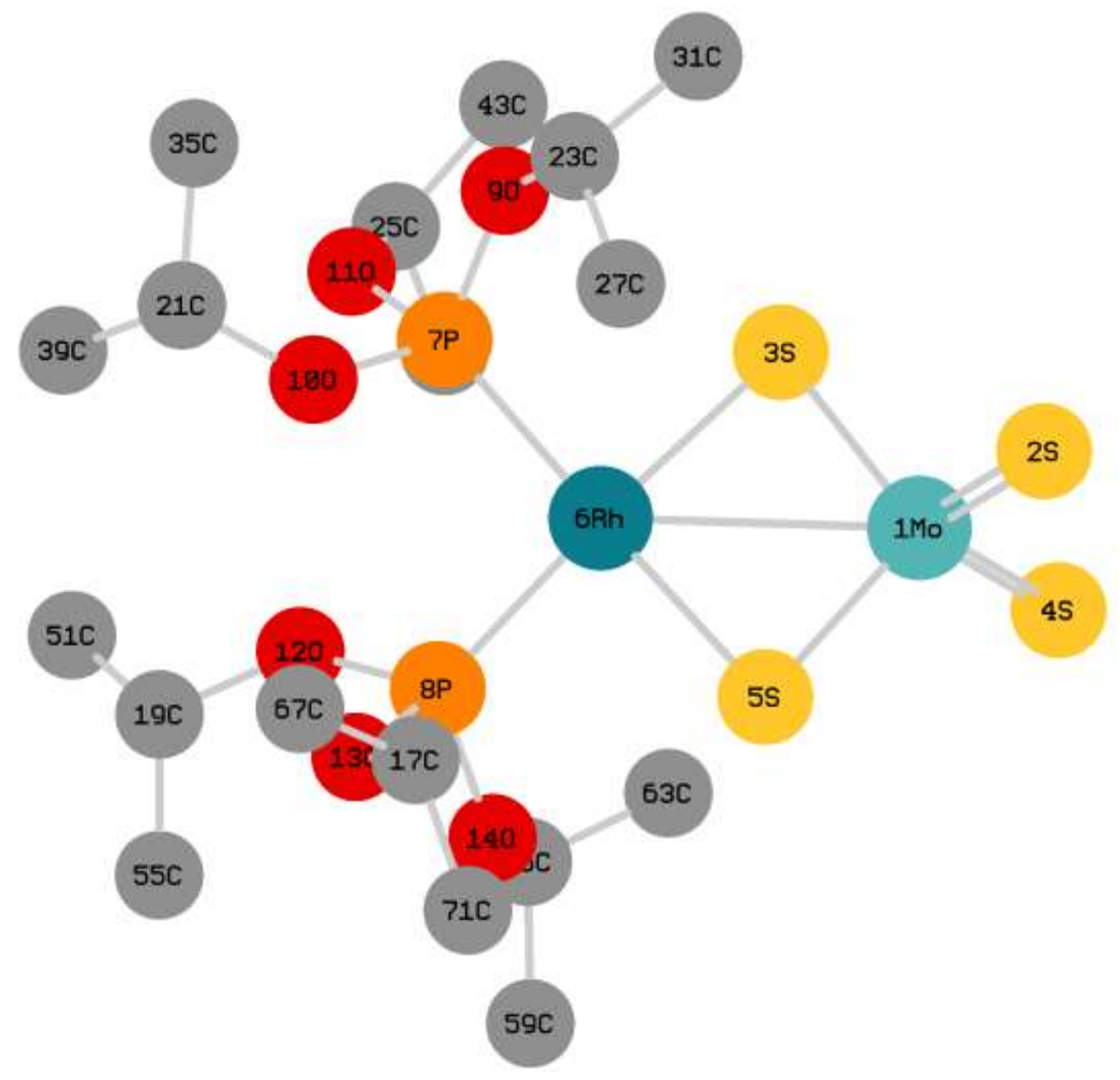

\begin{tabular}{|l|l|}
\hline \multicolumn{2}{|l|}{ Bond Lengths $(\mathbf{\AA})$} \\
\hline $7 \mathrm{P}-6 \mathrm{Rh}$ & 2.225 \\
\hline $8 \mathrm{P}-6 \mathrm{Rh}$ & 2.225 \\
\hline $6 \mathrm{Rh}-3 \mathrm{~S}$ & 2.402 \\
\hline $6 \mathrm{Rh}-5 \mathrm{~S}$ & 2.411 \\
\hline $1 \mathrm{Mo}-3 \mathrm{~S}$ & 2.252 \\
\hline $1 \mathrm{Mo}-5 \mathrm{~S}$ & 2.249 \\
\hline $6 \mathrm{Rh}-1 \mathrm{Mo}$ & 2.986 \\
\hline $1 \mathrm{Mo}-2 \mathrm{~S}$ & 2.163 \\
\hline $1 \mathrm{Mo}-4 \mathrm{~S}$ & 2.162 \\
\hline
\end{tabular}

\begin{tabular}{|l|l|}
\hline \multicolumn{2}{|l|}{ Bond Angles (deg) } \\
\hline 7P-6Rh-8P & 95.58 \\
\hline 6Rh-5S-1Mo & 79.74 \\
\hline 5S-1Mo-3S & 104.07 \\
\hline 1Mo-3S-6Rh & 79.61 \\
\hline 3S-6Rh-5S & 95.74 \\
\hline 2S-1Mo-4S & 110.36 \\
\hline
\end{tabular}




\section{Cartesian coordinates of all computed structures}

\begin{tabular}{|c|c|c|c|}
\hline \multicolumn{4}{|c|}{$\mathrm{E}(\mathrm{RB} 3 \mathrm{LYP})=-2084.28245843$ a.u. } \\
\hline $\mathrm{C}$ & 2.32576400 & 1.19264700 & -1.09502000 \\
\hline $\mathrm{H}$ & 1.75074200 & 1.85548500 & -1.73036300 \\
\hline $\mathrm{C}$ & 2.45911200 & -0.13289300 & -1.52503800 \\
\hline $\mathrm{H}$ & 1.95873200 & -0.39502200 & -2.44834200 \\
\hline $\mathrm{C}$ & 3.59040400 & -1.06220300 & -1.11477300 \\
\hline $\mathrm{H}$ & 3.82393300 & -1.72796700 & -1.94853500 \\
\hline $\mathrm{H}$ & 4.49833200 & -0.48435800 & -0.93176400 \\
\hline $\mathrm{C}$ & 3.23476300 & 1.90817900 & -0.12110300 \\
\hline $\mathrm{H}$ & 4.14782300 & 2.25390000 & -0.62657400 \\
\hline $\mathrm{H}$ & 2.71172300 & 2.80838300 & 0.20723300 \\
\hline $\mathrm{C}$ & 3.23476800 & -1.90817600 & 0.12110500 \\
\hline $\mathrm{H}$ & 2.71173000 & -2.80838100 & -0.20723100 \\
\hline $\mathrm{H}$ & 4.14782800 & -2.25389400 & 0.62657600 \\
\hline $\mathrm{C}$ & 3.59040100 & 1.06220600 & 1.11477400 \\
\hline $\mathrm{H}$ & 4.49833000 & 0.48436300 & 0.93176500 \\
\hline $\mathrm{H}$ & 3.82392800 & 1.72797000 & 1.94853700 \\
\hline $\mathrm{C}$ & 2.32576600 & -1.19264600 & 1.09502100 \\
\hline $\mathrm{H}$ & 1.75074400 & -1.85548600 & 1.73036300 \\
\hline $\mathrm{C}$ & 2.45911000 & 0.13289400 & 1.52503800 \\
\hline $\mathrm{H}$ & 1.95872800 & 0.39502300 & 2.44834100 \\
\hline $\mathrm{Rh}$ & 0.89096800 & -0.00000100 & -0.00000100 \\
\hline Mo & -2.09860000 & 0.00000000 & 0.00000000 \\
\hline $\mathrm{S}$ & -0.69579000 & -0.46701800 & 1.69962000 \\
\hline S & -0.69579100 & 0.46701900 & -1.69962000 \\
\hline $\mathrm{S}$ & -3.33719200 & -1.70235800 & -0.47203500 \\
\hline $\mathrm{S}$ & -3.33719400 & 1.70235700 & 0.47203500 \\
\hline
\end{tabular}

$\left[(\mathrm{CO})_{2} \mathrm{Rh}\left(\mathrm{MoS}_{4}\right)\right]^{-} \mathbf{3}$

$\mathrm{E}(\mathrm{RB} 3 \mathrm{LYP})=-1998.91383401$ a.u.

$\begin{array}{lcrc}\text { Mo } & 1.51507500 & -0.00002500 & -0.00004600 \\ \text { S } & -2.75658800 & 0.00009100 & 1.75616100 \\ \text { S } & -0.13691100 & -1.78914700 & -0.00028900 \\ \text { S } & -2.75850100 & -0.00013400 & -1.75492000 \\ \text { S } & -0.13689500 & 1.78903000 & -0.00055500 \\ \text { Rh } & 1.47913700 & -0.00014800 & -0.00012600 \\ \text { C } & 2.74780000 & 1.36511100 & 0.00001800 \\ \text { C } & 2.74813700 & -1.36507000 & -0.00002800 \\ \text { O } & 3.54571500 & -2.18681300 & 0.00005900 \\ \text { O } & 3.54411800 & 2.18807100 & 0.00010900\end{array}$

$\left[\left(\mathrm{P}(\mathrm{OPh})_{3}\right)_{2} \mathrm{Rh}\left(\mathrm{MoS}_{4}\right)\right]^{-} \quad 4$

$E(R B 3 L Y P)=-4296.99027497$ a.u.

Mo $\begin{array}{llll}3.84329400 & -0.01868000 & -0.00385600\end{array}$ 


\begin{tabular}{|c|c|c|c|}
\hline S & 5.06745600 & -1.78144200 & -0.21408900 \\
\hline $\mathrm{S}$ & 2.47379800 & -0.20997400 & 1.77327800 \\
\hline S & 5.08810000 & 1.72992800 & 0.20324200 \\
\hline S & 2.47187600 & 0.18793200 & -1.77780300 \\
\hline $\mathrm{Rh}$ & 0.85633600 & -0.00273800 & -0.00047500 \\
\hline$P$ & -0.53693500 & -0.76372900 & 1.53155900 \\
\hline$P$ & -0.53215000 & 0.77113300 & -1.53020200 \\
\hline $\mathrm{O}$ & -0.24613500 & -2.22999400 & 2.18423200 \\
\hline $\mathrm{O}$ & -2.10227100 & -0.94293500 & 1.07334200 \\
\hline $\mathrm{O}$ & -0.69491400 & -0.06971500 & 3.00347000 \\
\hline $\mathrm{O}$ & -2.09492100 & 0.96490400 & -1.06913300 \\
\hline $\mathrm{O}$ & -0.69927400 & 0.07859600 & -3.00176200 \\
\hline $\mathrm{O}$ & -0.22851500 & 2.23438200 & -2.18388400 \\
\hline $\mathrm{C}$ & 0.03359900 & 3.39963600 & -1.47331000 \\
\hline $\mathrm{C}$ & 1.30795300 & 3.65449700 & -0.98161000 \\
\hline $\mathrm{C}$ & -0.98290600 & 4.34039200 & -1.34608900 \\
\hline $\mathrm{C}$ & 1.55535300 & 4.86365500 & -0.34225900 \\
\hline $\mathrm{H}$ & 2.08769700 & 2.91626700 & -1.09558100 \\
\hline $\mathrm{C}$ & -0.71865600 & 5.54998700 & -0.71295500 \\
\hline $\mathrm{H}$ & -1.96191100 & 4.12464400 & -1.74961300 \\
\hline $\mathrm{C}$ & 0.54912900 & 5.81405900 & -0.20620400 \\
\hline $\mathrm{H}$ & 2.54511300 & 5.05197200 & 0.05159200 \\
\hline $\mathrm{H}$ & -1.50844100 & 6.28458000 & -0.61805900 \\
\hline $\mathrm{H}$ & 0.75184200 & 6.75384000 & 0.29096400 \\
\hline $\mathrm{C}$ & -3.19329100 & 1.47888100 & -1.71744500 \\
\hline $\mathrm{C}$ & -4.31197100 & 1.70095900 & -0.91375600 \\
\hline $\mathrm{C}$ & -3.24746100 & 1.76601500 & -3.08083600 \\
\hline $\mathrm{C}$ & -5.47812800 & 2.20809800 & -1.46859100 \\
\hline $\mathrm{H}$ & -4.24909000 & 1.46873300 & 0.13985200 \\
\hline $\mathrm{C}$ & -4.42442000 & 2.27478000 & -3.62160700 \\
\hline $\mathrm{H}$ & -2.38716000 & 1.59753400 & -3.70705600 \\
\hline $\mathrm{C}$ & -5.54225100 & 2.49941200 & -2.82763400 \\
\hline $\mathrm{H}$ & -6.33843500 & 2.37591100 & -0.83343500 \\
\hline $\mathrm{H}$ & -4.45919100 & 2.49685000 & -4.68055200 \\
\hline $\mathrm{H}$ & -6.45088300 & 2.89592400 & -3.26087700 \\
\hline $\mathrm{C}$ & -0.58305900 & -1.26509700 & -3.29078300 \\
\hline $\mathrm{C}$ & -1.22371700 & -2.24301400 & -2.53957400 \\
\hline $\mathrm{C}$ & 0.15879800 & -1.60532500 & -4.41587600 \\
\hline $\mathrm{C}$ & -1.11059000 & -3.57532500 & -2.92183700 \\
\hline $\mathrm{H}$ & -1.79221100 & -1.96799700 & -1.66319800 \\
\hline $\mathrm{C}$ & 0.25480700 & -2.93730100 & -4.79367100 \\
\hline $\mathrm{H}$ & 0.66277000 & -0.82372400 & -4.96614800 \\
\hline $\mathrm{C}$ & -0.37805100 & -3.92748200 & -4.04865200 \\
\hline $\mathrm{H}$ & -1.59071200 & -4.33830700 & -2.32416100 \\
\hline $\mathrm{H}$ & 0.84022900 & -3.20224000 & -5.66459100 \\
\hline $\mathrm{H}$ & -0.28996500 & -4.96648800 & -4.33727200 \\
\hline $\mathrm{C}$ & -3.20327900 & -1.44972000 & 1.72289300 \\
\hline $\mathrm{C}$ & -4.32321400 & -1.66783600 & 0.91989800 \\
\hline $\mathrm{C}$ & -3.25885700 & -1.73304200 & 3.08701900 \\
\hline $\mathrm{C}$ & -5.49197600 & -2.16743400 & 1.47611200 \\
\hline $\mathrm{H}$ & -4.25926000 & -1.43862800 & -0.13430400 \\
\hline $\mathrm{C}$ & -4.43840600 & -2.23425900 & 3.62919300 \\
\hline $\mathrm{H}$ & -2.39758200 & -1.56753800 & 3.71269600 \\
\hline $\mathrm{C}$ & -5.55748100 & -2.45503100 & 2.83588000 \\
\hline $\mathrm{H}$ & -6.35320800 & -2.33234000 & 0.84145100 \\
\hline $\mathrm{H}$ & -4.47421900 & -2.45348500 & 4.68869600 \\
\hline $\mathrm{H}$ & -6.46813000 & -2.84571100 & 3.27018600 \\
\hline $\mathrm{C}$ & -0.56034600 & 1.27223300 & 3.29278600 \\
\hline $\mathrm{C}$ & 0.18963500 & 1.60245000 & 4.41544900 \\
\hline $\mathrm{C}$ & -1.19138500 & 2.25844300 & 2.54437200 \\
\hline
\end{tabular}




$\begin{array}{lrrr}\mathrm{C} & 0.30367700 & 2.93291100 & 4.79358800 \\ \mathrm{H} & 0.68571300 & 0.81428400 & 4.96351500 \\ \mathrm{C} & -1.06028000 & 3.58901900 & 2.92694300 \\ \mathrm{H} & -1.76639300 & 1.99099100 & 1.66992000 \\ \mathrm{C} & -0.31939500 & 3.93132800 & 4.05133900 \\ \mathrm{H} & 0.89544600 & 3.19001000 & 5.66256000 \\ \mathrm{H} & -1.53300100 & 4.35827900 & 2.33142600 \\ \mathrm{H} & -0.21739800 & 4.96898400 & 4.34023600 \\ \mathrm{C} & 0.00217400 & -3.39795000 & 1.47309500 \\ \mathrm{C} & 1.27085400 & -3.66334500 & 0.97236900 \\ \mathrm{C} & -1.02241300 & -4.33105700 & 1.35461800 \\ \mathrm{C} & 1.50415700 & -4.87518700 & 0.33278000 \\ \mathrm{H} & 2.05738700 & -2.93144400 & 1.08005200 \\ \mathrm{C} & -0.77223200 & -5.54343700 & 0.72109200 \\ \mathrm{H} & -1.99673100 & -4.10740400 & 1.76509800 \\ \mathrm{C} & 0.48968200 & -5.81795100 & 0.20530000 \\ \mathrm{H} & 2.48962500 & -5.07166800 & -0.06778900 \\ \mathrm{H} & -1.56836200 & -6.27200400 & 0.63303200 \\ \mathrm{H} & 0.68150700 & -6.75991600 & -0.29205600\end{array}$

$\left[\left(\mathrm{P}(\mathrm{OMe})_{3}\right)_{2} \mathrm{Rh}\left(\mathrm{MoS}_{4}\right)\right]^{-} \mathbf{6}$

$E(R B 3 L Y P)=-3146.13686496$ a.u.

Mo $\quad-2.75717300 \quad-0.00000900 \quad-0.00000200$

S $\quad-3.99452200 \quad 0.81681700 \quad-1.57148300$

$\begin{array}{llll}\mathrm{S} & -1.38732300 & 1.57800300 & 0.84097500\end{array}$

$\begin{array}{llll}\mathrm{S} & -3.99451900 & -0.81684100 & 1.57147800\end{array}$

S $\quad-1.38731500 \quad-1.57801300 \quad-0.84098100$

$\begin{array}{llll}\mathrm{Rh} & 0.22629800 & -0.00000200 & 0.00000000\end{array}$

$\begin{array}{llll}\mathrm{P} & 1.74628100 & 1.59718900 & 0.10745400\end{array}$

$\begin{array}{llll}\mathrm{P} & 1.74629700 & -1.59718000 & -0.10745200\end{array}$

O $\quad 1.32432500 \quad 3.08627500 \quad-0.40260200$

O $\quad 3.07413000 \quad 1.31629500 \quad-0.77641800$

$\begin{array}{llll}\mathrm{O} & 2.46591500 & 2.05789900 & 1.48654500\end{array}$

$\begin{array}{llll}\mathrm{O} & 3.07415000 & -1.31626300 & 0.77640500\end{array}$

O $\quad 2.46594300 \quad-2.05788500 \quad-1.48653900$

O $\quad 1.32437300 \quad-3.08626700 \quad 0.40262600$

C $\quad 0.54845800 \quad-3.26761000 \quad 1.59223400$

$\begin{array}{llll}\mathrm{H} & 0.42807200 & -4.34246300 & 1.71769100\end{array}$

$\mathrm{H} \quad \begin{array}{llll}1.06722300 & -2.85773600 & 2.46182200\end{array}$

$\mathrm{H} \quad-0.42843200 \quad-2.79797300 \quad 1.49073900$

$\begin{array}{llll}\text { C } & 1.70729900 & -2.23327500 & -2.68424300\end{array}$

$\mathrm{H} \quad 1.16239100 \quad-3.17778400 \quad-2.65612600$

$\mathrm{H} \quad 0.99602700 \quad-1.41787000 \quad-2.81685400$

$\mathrm{H} \quad 2.42024500 \quad-2.24466200 \quad-3.50735500$

$\begin{array}{llll}\mathrm{C} & 4.19467400 & -2.19874100 & 0.78144000\end{array}$

$\mathrm{H} \quad 4.83798500 \quad-1.88233000 \quad 1.60117200$

$\mathrm{H} \quad 3.88337000 \quad-3.23236800 \quad 0.93972000$

$\mathrm{H} \quad 4.73984500 \quad-2.12947800 \quad-0.16022300$

$\begin{array}{llll}\text { C } & 4.19464200 & 2.19878900 & -0.78146700\end{array}$

$\mathrm{H} \quad 3.88332100 \quad 3.23241000 \quad-0.93975300$

$\mathrm{H} \quad 4.73982000 \quad 2.12954200 \quad 0.16019300$

$\mathrm{H} \quad 4.83795100 \quad 1.88238100 \quad-1.60120100$

C $\quad 0.54839000 \quad 3.26761400 \quad-1.59219600$

$\mathrm{H} \quad-0.42849600 \quad 2.79797000 \quad-1.49068700$

$\mathrm{H} \quad 0.42799600 \quad 4.34246600 \quad-1.71765100$

$\mathrm{H} \quad \begin{array}{llll}\mathrm{H} & 1.06714500 & 2.85774200 & -2.46179100\end{array}$

$\begin{array}{llll}\mathrm{C} & 1.70726200 & 2.23327400 & 2.68424500\end{array}$ 


$\begin{array}{llll}\mathrm{H} & 2.42020200 & 2.24467300 & 3.50736100 \\ \mathrm{H} & 1.16233400 & 3.17777200 & 2.65612700 \\ \mathrm{H} & 0.99600500 & 1.41785400 & 2.81684800\end{array}$

$\left[\left(\mathrm{P}(\mathrm{OEt})_{3}\right)_{2} \mathrm{Rh}\left(\mathrm{MoS}_{4}\right)\right]^{-} \mathbf{7}$

$\mathrm{E}(\mathrm{RB} 3 \mathrm{LYP})=-3382.14140952$ a.u.

$\begin{array}{llll}\text { Mo } & 3.13782200 & 0.00007400 & 0.00006500\end{array}$

$\begin{array}{llll}\mathrm{S} & 4.37600900 & 0.87964900 & 1.53694800\end{array}$

S $\quad 1.76653000 \quad 1.54158200 \quad-0.90225500$

S $\quad 4.37666700 \quad-0.87927500 \quad-1.53641900$

$\begin{array}{llll}\mathrm{S} & 1.76651800 & -1.54159700 & 0.90208900\end{array}$

$\begin{array}{llll}\mathrm{Rh} & 0.14981400 & 0.00000200 & -0.00013000\end{array}$

$\begin{array}{lllll}\mathrm{P} & -1.37453300 & 1.58845800 & -0.21436000\end{array}$

$\begin{array}{lllll}\mathrm{P} & -1.37436900 & -1.58861900 & 0.21417800\end{array}$

$\begin{array}{llll}\mathrm{O} & -0.94517500 & 3.11205000 & 0.16734300\end{array}$

$\begin{array}{llll}\mathrm{O} & -2.69720700 & 1.37750400 & 0.69851500\end{array}$

$\begin{array}{lllll}\mathrm{O} & -2.09897800 & 1.93224200 & -1.62496500\end{array}$

$\begin{array}{llll}\mathrm{O} & -2.69747600 & -1.37754900 & -0.69806800\end{array}$

$\begin{array}{llll}\mathrm{O} & -2.09821000 & -1.93300000 & 1.62495400\end{array}$

O $\quad-0.94501400 \quad-3.11200700 \quad-0.16825500$

C $\quad-0.18639500 \quad-3.40809700 \quad-1.35472100$

$\mathrm{H} \quad-0.87695500 \quad-3.45629700 \quad-2.20256200$

$\mathrm{H} \quad 0.53357800 \quad-2.61058200 \quad-1.53949800$

C $\quad-1.34860100 \quad-1.97741400 \quad 2.84920100$

$\mathrm{H} \quad-0.77470900 \quad-2.90621500 \quad 2.87828400$

$\mathrm{H} \quad-0.64014000 \quad-1.14765600 \quad 2.86682300$

C $\quad-3.82283600 \quad-2.26452000 \quad-0.65533200$

$\mathrm{H} \quad-4.37366500 \quad-2.09052300 \quad 0.27105600$

$\mathrm{H} \quad-3.47465100 \quad-3.29953500 \quad-0.64884100$

$\begin{array}{llll}\mathrm{C} & -3.82268000 & 2.26437200 & 0.65605200\end{array}$

$\mathrm{H} \quad-3.47458200 \quad 3.29940400 \quad 0.64833300$

$\mathrm{H} \quad-4.37433000 \quad 2.08951500 \quad-0.26968500$

$\begin{array}{llll}\mathrm{C} & -0.18677300 & 3.40855400 & 1.35383100\end{array}$

$\mathrm{H} \quad 0.53326300 \quad 2.61117200 \quad 1.53891600$

$\mathrm{H} \quad-0.87742900 \quad 3.45693800 \quad 2.20159300$

$\begin{array}{llll}\text { C } & -1.34949400 & 1.97740900 & -2.84926600\end{array}$

$\mathrm{H} \quad-0.77696800 \quad 2.90704900 \quad-2.87859400$

$\mathrm{H} \quad-0.63984100 \quad 1.14865800 \quad-2.86670300$

C $\quad-2.32147900 \quad-1.90099500 \quad 4.00728400$

$\mathrm{H} \quad-3.03100400 \quad-2.72950200 \quad 3.97758900$

$\mathrm{H} \quad-1.77698300 \quad-1.94818600 \quad 4.95239300$

$\mathrm{H} \quad-2.88186200 \quad-0.96606400 \quad 3.97809400$

$\begin{array}{llll}\mathrm{C} & 0.52440400 & -4.72898900 & -1.15297900\end{array}$

$\mathrm{H} \quad-0.18676100 \quad-5.52843000 \quad-0.93691200$

$\mathrm{H} \quad 1.08067500 \quad-4.99241900 \quad-2.05458200$

$\mathrm{H} \quad 1.22901100 \quad-4.64965200 \quad-0.32608100$

C $\quad-4.69273400 \quad-1.98920100 \quad-1.86546900$

$\mathrm{H} \quad-5.02501800 \quad-0.95088500 \quad-1.87280300$

$\mathrm{H} \quad-4.14351100 \quad-2.17658000 \quad-2.78868000$

$\mathrm{H} \quad-5.57349000 \quad-2.63507700 \quad-1.85047100$

$\begin{array}{llll}\mathrm{C} & -4.69144500 & 1.99004400 & 1.86723200\end{array}$

$\begin{array}{llll}\mathrm{H} & -5.57231100 & 2.63577400 & 1.85245300\end{array}$

$\begin{array}{llll}\mathrm{H} & -5.02356300 & 0.95168500 & 1.87580900\end{array}$

$\mathrm{H} \quad-4.14139300 \quad 2.17835300 \quad 2.78975800$

$\begin{array}{llll}\mathrm{C} & -2.32230800 & 1.89928100 & -4.00729400\end{array}$

$\mathrm{H} \quad-3.03310200 \quad 2.72670300 \quad-3.97769100$

$\mathrm{H} \quad-1.77794100 \quad 1.94714800 \quad-4.95244300$ 


$\begin{array}{lrrr}\mathrm{H} & -2.88124500 & 0.96349600 & -3.97788600 \\ \mathrm{C} & 0.52395600 & 4.72944600 & 1.15182900 \\ \mathrm{H} & 1.08003200 & 4.99316100 & 2.05346800 \\ \mathrm{H} & 1.22872900 & 4.64997300 & 0.32508500 \\ \mathrm{H} & -0.18724000 & 5.52877000 & 0.93543000\end{array}$

$\left[\left(\mathrm{P}(\mathrm{O}-i-\mathrm{Et})_{3}\right)_{2} \mathrm{Rh}\left(\mathrm{MoS}_{4}\right)\right]^{-} \mathbf{8}$

$\mathrm{E}(\mathrm{RB} 3 \mathrm{LYP})=-3618.10359575$ a.u.

$\begin{array}{lrrr}\text { Mo } & 3.30352500 & -0.34620700 & 0.00642600 \\ \text { S } & 4.60783500 & 0.65192200 & 1.41498100 \\ \text { S } & 2.09253100 & 1.15570500 & -1.15600300 \\ \text { S } & 4.45783400 & -1.57515100 & -1.34690900 \\ \text { S } & 1.78423100 & -1.58329300 & 1.11158300 \\ \text { Rh } & 0.33502700 & -0.02591800 & -0.02255300 \\ \text { P } & -0.98161000 & 1.75892900 & -0.20393400 \\ \text { P } & -1.31370100 & -1.48390900 & 0.24096200 \\ \text { O } & -0.28271800 & 3.20347400 & 0.01911800 \\ \text { O } & -2.17917300 & 1.74556700 & 0.89366200 \\ \mathrm{O} & -1.86423200 & 2.15643000 & -1.50994000 \\ \mathrm{O} & -2.61543900 & -1.25631800 & -0.70678900 \\ \mathrm{O} & -2.05788400 & -1.72352400 & 1.66300000 \\ \mathrm{O} & -0.94404000 & -3.03724200 & -0.07448400 \\ \mathrm{C} & -0.70589400 & -3.61557200 & -1.37851400 \\ \mathrm{H} & -1.51285800 & -3.28281500 & -2.03834100 \\ \mathrm{C} & -1.43073800 & -1.47607900 & 2.94341000 \\ \mathrm{H} & -0.53494200 & -0.87721600 & 2.76971300 \\ \mathrm{C} & -3.96968300 & -1.70638800 & -0.48322100 \\ \mathrm{H} & -4.31175200 & -1.24918900 & 0.44787000 \\ \mathrm{C} & -3.33336600 & 2.60188100 & 1.07690300 \\ \mathrm{H} & -3.54789600 & 2.48900700 & 2.14320500 \\ \mathrm{C} & 0.45825100 & 3.76105300 & 1.13525100 \\ \mathrm{H} & -0.22095100 & 4.50272700 & 1.56968900 \\ \mathrm{C} & -1.49193900 & 2.20920600 & -2.91006100 \\ \mathrm{H} & -2.41677400 & 2.56309600 & -3.37421500 \\ \mathrm{C} & 0.84295100 & 2.77535600 & 2.22910400 \\ \mathrm{H} & -0.02379600 & 2.22979300 & 2.60024300 \\ \mathrm{H} & 1.58859700 & 2.06027400 & 1.88947800 \\ \mathrm{H} & 1.27019800 & 3.34021800 & 3.06093200 \\ \mathrm{C} & 1.66337900 & 4.48782300 & 0.55866200 \\ \mathrm{H} & 2.35474200 & 3.77479700 & 0.11139900 \\ \mathrm{H} & 1.34999200 & 5.20094900 & -0.20479100 \\ \mathrm{H} & 2.18164000 & 5.03234200 & 1.35058800 \\ \mathrm{C} & -3.09827100 & 4.08215700 & 0.80071500 \\ \mathrm{H} & -2.29201800 & 4.48116600 & 1.41190600 \\ \mathrm{H} & -2.86062800 & 4.25967500 & -0.24570600 \\ \mathrm{H} & -4.01099400 & 4.62862600 & 1.04969000 \\ \mathrm{C} & -4.52131600 & 2.06007900 & 0.29389700 \\ \mathrm{H} & -5.42428900 & 2.61439300 & 0.56017000 \\ \mathrm{H} & -4.35247700 & 2.15795900 & -0.77689000 \\ \mathrm{H} & -4.68232600 & 1.00971200 & 0.52746700 \\ \mathrm{C} & -0.39987600 & 3.23583000 & -3.17677900 \\ \mathrm{H} & -0.27326900 & 3.35203700 & -4.25553300 \\ \mathrm{H} & -0.66610400 & 4.20336700 & -2.75210300 \\ \mathrm{H} & 0.55294100 & 2.92129400 & -2.75320500 \\ \mathrm{C} & -1.17066500 & 0.83314400 & -3.47699100 \\ \mathrm{H} & -0.21991500 & 0.45905100 & -3.10233200 \\ \mathrm{H} & -1.94947700 & 0.11950900 & -3.21231800 \\ & & & \end{array}$




$\begin{array}{lrrr}\mathrm{H} & -1.11172600 & 0.90143200 & -4.56594200 \\ \mathrm{C} & -4.78482200 & -1.17029500 & -1.65070600 \\ \mathrm{H} & -5.84436000 & -1.39073500 & -1.50610700 \\ \mathrm{H} & -4.66184600 & -0.09372300 & -1.74615400 \\ \mathrm{H} & -4.46134300 & -1.63646400 & -2.58310500 \\ \mathrm{C} & -4.10135400 & -3.21954100 & -0.35625900 \\ \mathrm{H} & -3.77612800 & -3.71635600 & -1.27135300 \\ \mathrm{H} & -3.51647700 & -3.60111700 & 0.47613500 \\ \mathrm{H} & -5.15100000 & -3.47271300 & -0.18911300 \\ \mathrm{C} & -0.80656100 & -5.12208900 & -1.18306600 \\ \mathrm{H} & -1.77095400 & -5.39956800 & -0.75753000 \\ \mathrm{H} & -0.02105100 & -5.46289900 & -0.50734700 \\ \mathrm{C} & 0.63013300 & -3.20742900 & -1.97919000 \\ \mathrm{H} & 0.68373900 & -2.13487300 & -2.15552000 \\ \mathrm{H} & 1.45699800 & -3.46908300 & -1.32073900 \\ \mathrm{H} & 0.76403700 & -3.71839400 & -2.93583000 \\ \mathrm{C} & -2.41929800 & -0.68905300 & 3.78783100 \\ \mathrm{H} & -2.66683100 & 0.25177900 & 3.29799900 \\ \mathrm{H} & -3.33880400 & -1.25962500 & 3.93760500 \\ \mathrm{H} & -1.98826900 & -0.46903200 & 4.76627900 \\ \mathrm{C} & -1.03386700 & -2.80533300 & 3.56622600 \\ \mathrm{H} & -0.33009300 & -3.33030600 & 2.92277000 \\ \mathrm{H} & -0.55343500 & -2.63813800 & 4.53213000 \\ \mathrm{H} & -1.91228500 & -3.43650600 & 3.71902300\end{array}$


SI.5. TDDFT predicted UV-Vis spectra of complexes 3, 6, 7 and 8.

$\left[(\mathrm{CO})_{2} \mathrm{Rh}\left(\mathrm{MoS}_{4}\right)\right]^{-} \mathbf{3}$

\section{Excitation energies and oscillator strengths:}

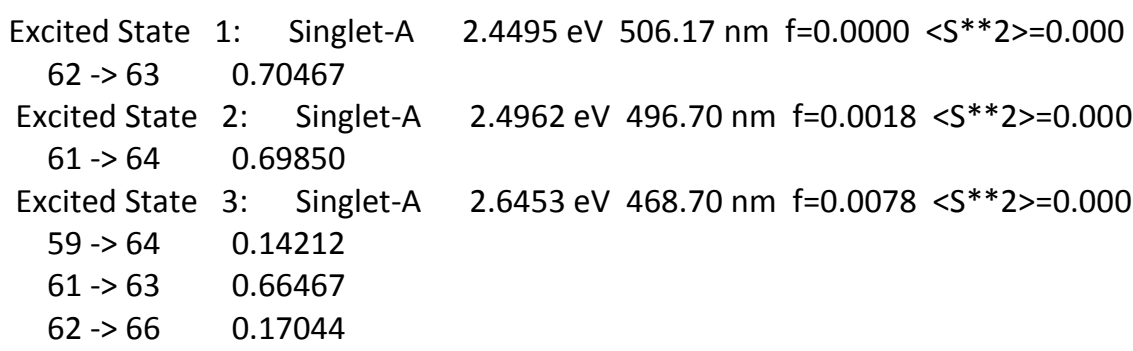

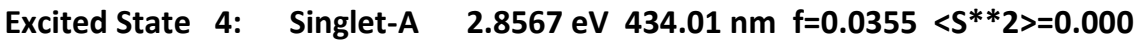
$57->63 \quad-0.11025$

$59 \rightarrow 66 \quad-0.10041$

62 -> $64 \quad 0.67701$

Excited State 5: $\quad$ Singlet-A $\quad 3.1217$ eV $397.16 \mathrm{~nm} \mathrm{f=0.0029}<\mathrm{S}^{* *} 2>=0.000$ $59 \rightarrow 64 \quad-0.18690$

$61->63 \quad-0.11781$

$62 \rightarrow 66 \quad 0.65973$

Excited State 6 : $\quad$ Singlet-A $\quad 3.1245 \mathrm{eV} \quad 396.82 \mathrm{~nm} \mathrm{f}=0.0000<\mathrm{S}^{* *} 2>=0.000$ $61>66 \quad 0.69396$

Excited State 7: $\quad$ Singlet-A $\quad 3.2165 \mathrm{eV} \quad 385.46 \mathrm{~nm} \mathrm{f}=0.0059<\mathrm{S}^{* *} 2>=0.000$ $59->63 \quad-0.13672$

$62 \rightarrow 65 \quad 0.67430$

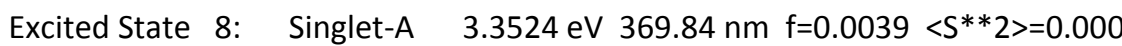
$59 \rightarrow 63 \quad 0.68751$ $62 \rightarrow 65 \quad 0.12571$

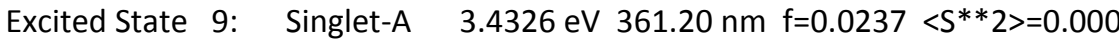
61 -> $65 \quad 0.69940$

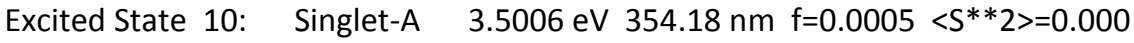
$60->63 \quad 0.69723$

Excited State 11: Singlet-A $3.5321 \mathrm{eV} \quad 351.02 \mathrm{~nm} \mathrm{f}=0.0015<\mathrm{S}^{* *} 2>=0.000$ $58 \rightarrow 63 \quad 0.33850$

$59->64 \quad 0.46821$

$61 \rightarrow 67 \quad 0.37486$

$61>70 \quad 0.11199$

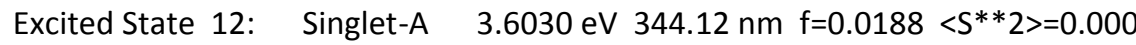
$58 \rightarrow 63-0.29727$

$59->64 \quad 0.11414$

$60>65 \quad 0.60006$

$61->67 \quad 0.11533$

Excited State 13: Singlet-A $\quad 3.6154$ eV $342.93 \mathrm{~nm} \mathrm{f=0.0014<S**} 2>=0.000$ $58 \rightarrow 63 \quad 0.52621$

$59->64 \quad-0.17962$

$60->65 \quad 0.34700$

$61->67 \quad-0.23366$

Excited State 14: Singlet-A 3.6395 eV $340.66 \mathrm{~nm} \mathrm{f}=0.0000<S^{* *} 2>=0.000$ $57->64 \quad-0.11596$ $60->64 \quad 0.69331$

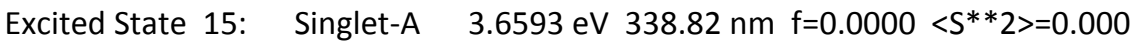
$62->67 \quad 0.68423$ 
62 -> $70 \quad 0.10878$

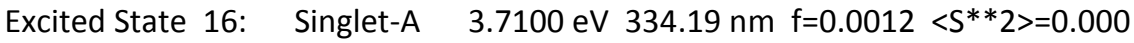

$58 \rightarrow 64 \quad-0.19839$

$60->66 \quad 0.63121$

$60 \rightarrow 68 \quad 0.16919$

$60->72 \quad 0.14768$

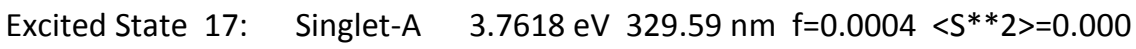
$58 \rightarrow 64 \quad 0.59389$

$60->66 \quad 0.19482$

$62->69 \quad 0.30252$

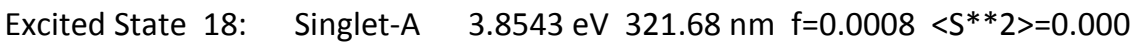
$59->66 \quad-0.14680$

$61->69 \quad 0.67259$

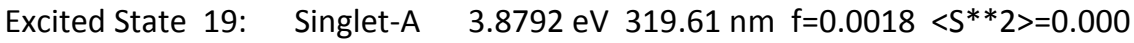
$55 \rightarrow 66 \quad 0.10055$

$59->64 \quad 0.14258$

$61 \rightarrow 67-0.18589$

$62->68 \quad 0.62933$

$62 \rightarrow 72 \quad 0.12065$

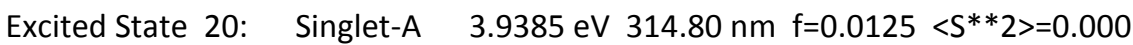
$53 \rightarrow 63 \quad-0.11108$

$56->66 \quad 0.10425$

$58->64 \quad-0.27364$

$62->65 \quad-0.10580$

$62 \rightarrow 69 \quad 0.61362$

$\left[\left(\mathrm{P}(\mathrm{OMe})_{3}\right)_{2} \mathrm{Rh}\left(\mathrm{MoS}_{4}\right)\right]^{-} \mathbf{6}$

Excitation energies and oscillator strengths:

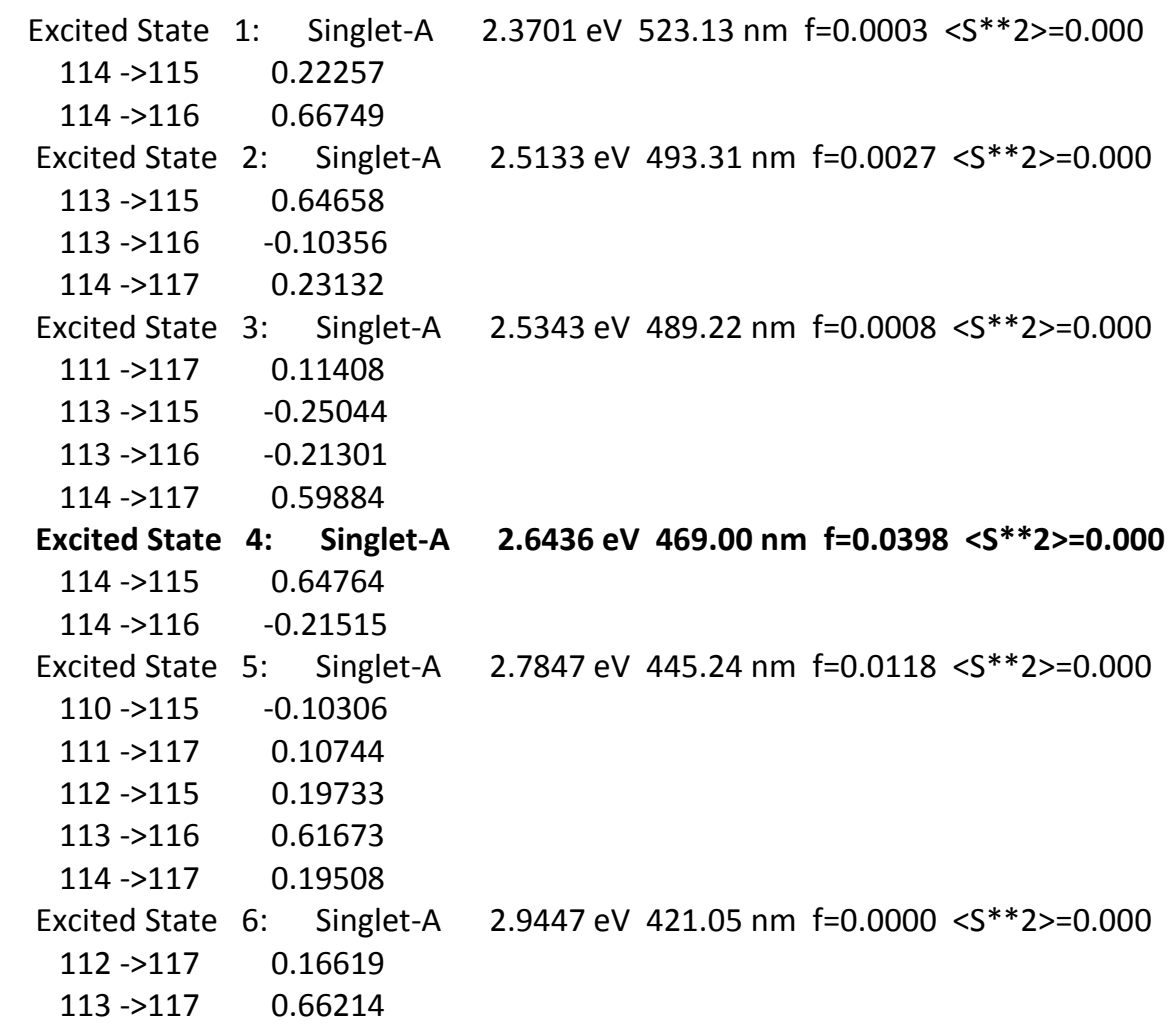


$113->118 \quad 0.10851$

Excited State 7: Singlet-A $\quad 3.2136 \mathrm{eV} 385.81 \mathrm{~nm} \mathrm{f}=0.0090<\mathrm{S} * * 2>=0.000$

$111->117 \quad 0.56451$

$112->115 \quad-0.11616$

$112->116 \quad-0.31360$

$114->117 \quad-0.13677$

$114->118 \quad-0.12397$

Excited State 8: Singlet-A $\quad 3.2425 \mathrm{eV} \quad 382.37 \mathrm{~nm} \mathrm{f}=0.0004<\mathrm{S}^{* *} 2>=0.000$

$111->117 \quad 0.31442$

$112->116 \quad 0.59746$

Excited State 9: $\quad$ Singlet-A $\quad 3.2504$ eV $381.44 \mathrm{~nm} \mathrm{f=0.0004}\left\langle\mathrm{S}^{* *} 2>=0.000\right.$

$111->115 \quad 0.23215$

$111->116 \quad 0.64158$

$114->119 \quad 0.12290$

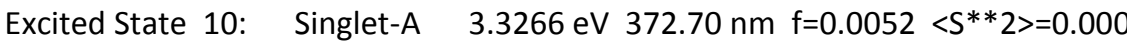
$109->117 \quad-0.10632$

$112 \rightarrow 115 \quad 0.63025$

$113->116 \quad-0.14276$

$113->119 \quad-0.12798$

$114->120 \quad 0.10215$

Excited State 11: $\quad$ Singlet-A $\quad 3.3524$ eV $369.84 \mathrm{~nm} \mathrm{f=0.0004}\left\langle\mathrm{S}^{* *} 2>=0.000\right.$ $111->115 \quad 0.57625$

$111->116 \quad-0.24820$

$112->117 \quad-0.26658$

$113->118 \quad 0.10990$

Excited State 12: Singlet-A $\quad 3.3689$ eV $368.02 \mathrm{~nm} \mathrm{f=0.0022}<\mathrm{S}^{* *} 2>=0.000$ $110->117 \quad 0.18511$

$111 \rightarrow 115 \quad 0.31429$

$112->117 \quad 0.52446$

$113->117 \quad-0.13720$

$113->118 \quad-0.17585$

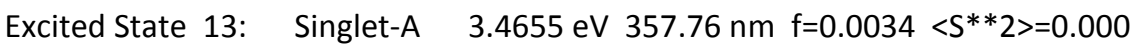
$109->117 \quad-0.15651$

$110->115 \quad-0.18599$

$110->116 \quad 0.15050$

$114->118 \quad 0.60782$

$114->120 \quad 0.10381$

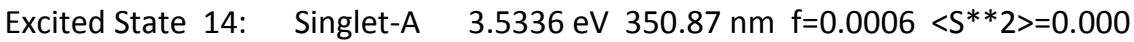
$110->115 \quad 0.36672$

$110->116 \quad 0.56848$

$113->119 \quad 0.12138$

Excited State 15: Singlet-A $\quad 3.5446$ eV $349.78 \mathrm{~nm} \mathrm{f=0.0011<S**} 2>=0.000$ $111->116 \quad-0.11427$

$114->119 \quad 0.67931$

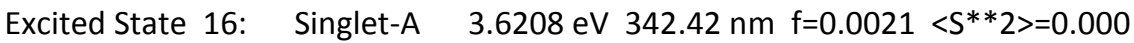

$110->115 \quad 0.16569$

$113->119 \quad 0.17084$

$114->120 \quad 0.64383$

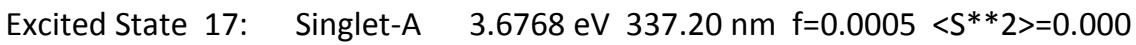
$109->115 \quad 0.65339$

$113->120 \quad-0.22632$

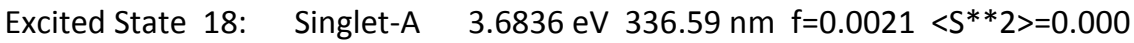

$110->115 \quad 0.34377$

$110->116 \quad-0.34190$

$112->116 \quad-0.11884$

$113->119 \quad 0.41628$

$114->118 \quad 0.11906$ 
$114->120 \quad-0.18656$

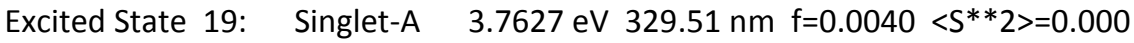

$109->116 \quad 0.57162$

$110->117 \quad 0.11588$

$113->120 \quad-0.34038$

Excited State 20: Singlet-A 3.7799 eV $328.01 \mathrm{~nm} \mathrm{f=0.0005}<\mathrm{S}^{* *} 2>=0.000$

$109->115 \quad-0.10793$

$109->116 \quad-0.20362$

$110->117 \quad-0.15641$

$112->117 \quad 0.17717$

$113->117 \quad-0.10616$

$113->118 \quad 0.50131$

$113->120 \quad-0.30327$

$113->122 \quad-0.10125$

Excited State 21: Singlet-A $3.8753 \mathrm{eV} 319.94 \mathrm{~nm} \mathrm{f=0.0096}<\mathrm{S}^{* *} 2>=0.000$ $108->117 \quad-0.21125$

$109->117 \quad 0.58340$

$114->118 \quad 0.19532$

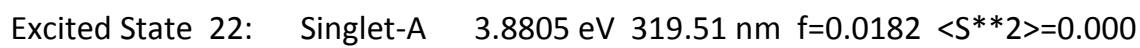
$108->115 \quad-0.12537$

$109->115 \quad 0.12714$

$109->116 \quad 0.14807$

$110->117 \quad 0.29752$

$113->117 \quad-0.13064$

$113->118 \quad 0.37910$

$113->120 \quad 0.40724$

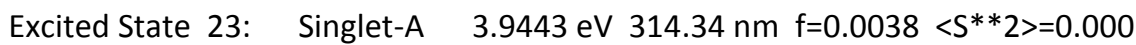
$107->117 \quad 0.18538$

$108->117 \quad 0.11002$

$110->115 \quad 0.19124$

$111>118 \quad 0.13505$

$113->119 \quad-0.23342$

$114->118 \quad 0.11107$

$114->122 \quad 0.52795$

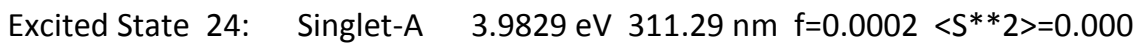
$107->115 \quad-0.13557$

$108->115 \quad 0.63927$

$110->117 \quad 0.15167$

$113->118 \quad 0.14100$

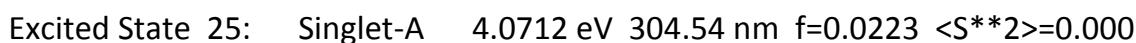
$106->115 \quad 0.10354$

$109->120 \quad 0.12694$

$110->115 \quad-0.25365$

$112->115 \quad 0.10767$

$112->119 \quad 0.16652$

$113->116 \quad-0.11065$

$113->119 \quad 0.38783$

$114->122 \quad 0.37162$

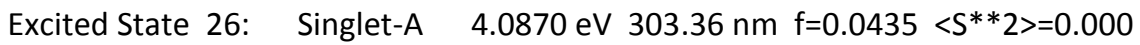
$107->116 \quad-0.10698$

$108->115 \quad-0.13281$

$108 \rightarrow 116 \quad 0.50735$

$109->116 \quad-0.19845$

$110->117 \quad 0.31117$

$112->117 \quad-0.12769$

$113->120 \quad-0.14029$

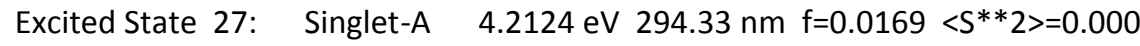




$\begin{array}{lr}107->115 & -0.18811 \\ 107->116 & 0.31871 \\ 108->116 & 0.36555 \\ 109->116 & 0.11056 \\ 110->117 & -0.31319 \\ 112->117 & 0.12644 \\ 112->118 & -0.18872\end{array}$

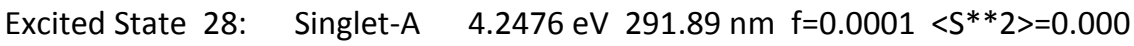
$106->116 \quad-0.21277$

$107->117 \quad 0.44945$

$108->117 \quad 0.36129$

$109->117 \quad 0.15556$

$111->118 \quad-0.10766$

$114->122 \quad-0.14412$

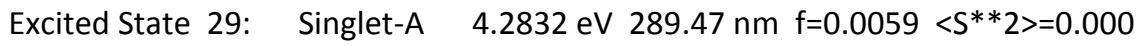
$106->115 \quad 0.13442$

$106->116 \quad 0.58525$

$108->117 \quad 0.11272$

$111->118 \quad-0.24849$

$112->119 \quad 0.11920$

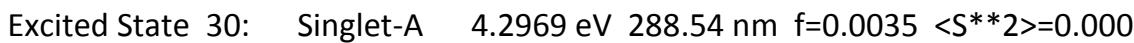
$107->116 \quad 0.38845$

$112->118 \quad 0.51195$

$112->120 \quad-0.15290$

$\left[\left(\mathrm{P}(\mathrm{OEt})_{3}\right)_{2} \mathrm{Rh}\left(\mathrm{MoS}_{4}\right)\right]^{-} \quad \mathbf{7}$

Excitation energies and oscillator strengths:

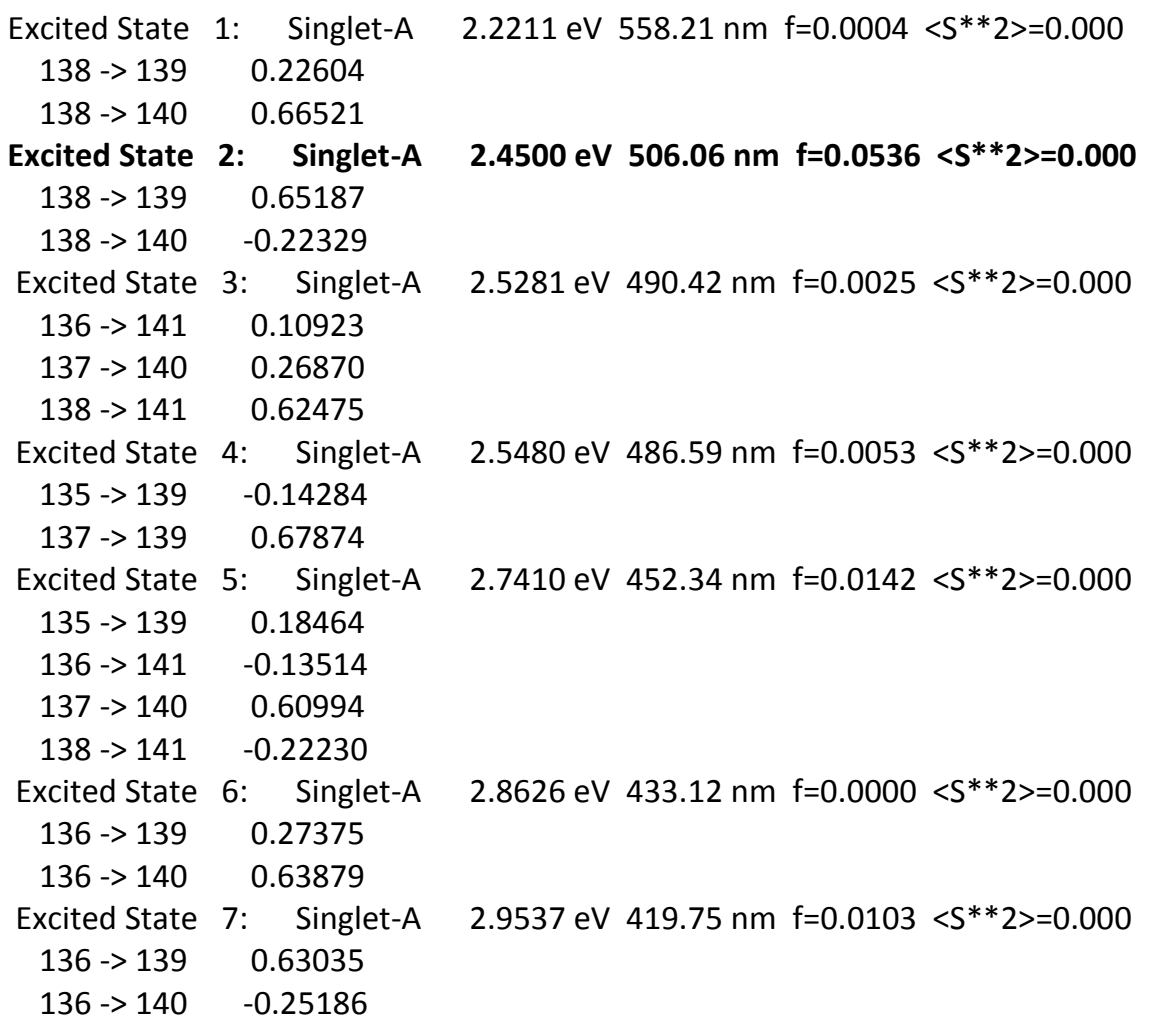


$137->141 \quad 0.15369$

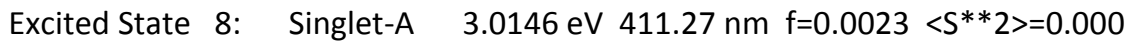

$135->141 \quad 0.18475$

$136->139 \quad-0.12893$

$137->141 \quad 0.63802$

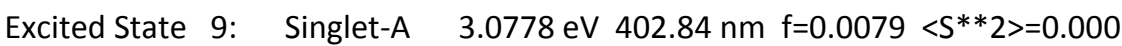

$135 \rightarrow 140 \quad 0.31286$

$136->141 \quad 0.56199$

$138->141-0.16323$

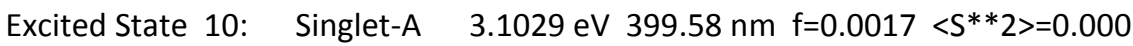

$135->139 \quad 0.52651$

$135->140 \quad-0.33594$

$136 \rightarrow 141 \quad 0.25921$

$137->139 \quad 0.15479$

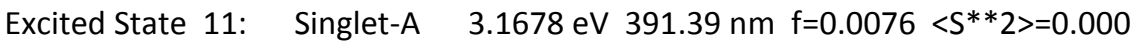

$133->139-0.18999$

$135->139 \quad 0.38401$

$135 \rightarrow 140 \quad 0.48400$

$136->141 \quad-0.16857$

$137->140 \quad-0.10653$

Excited State 12: Singlet-A $\quad 3.4037$ eV $364.27 \mathrm{~nm} \mathrm{f}=0.0025 \quad<\mathrm{S}^{* *} 2>=0.000$ $134->140 \quad 0.10208$

$135 \rightarrow 141-0.11814$

138 -> $143 \quad 0.67133$

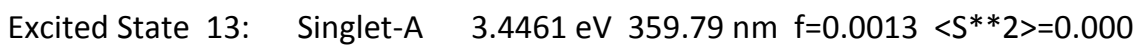
135 -> $141 \quad 0.58266$

$137 \rightarrow 141 \quad-0.20244$

$137->142 \quad-0.10176$

$137 \rightarrow 144 \quad 0.21581$

$138->143 \quad 0.13978$

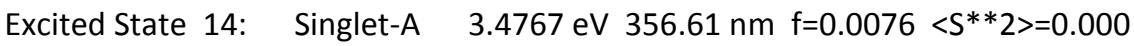

$133->139-0.15557$

$133 \rightarrow 140 \quad 0.11315$

$134->141 \quad 0.18478$

$138 \rightarrow 142 \quad 0.56209$

$138->144-0.27644$

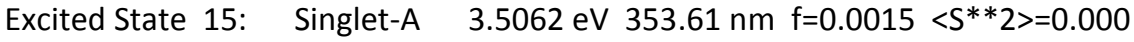

$133 \rightarrow 139 \quad 0.31996$

$133 \rightarrow 140 \quad 0.59579$

$137 \rightarrow 143 \quad 0.10761$

$138 \rightarrow 144 \quad 0.13082$

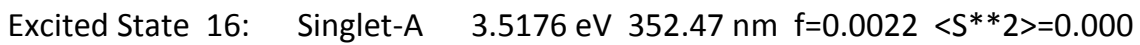

$133 \rightarrow 140-0.16126$

$134->139 \quad-0.28018$

$138 \rightarrow 142 \quad 0.33772$

$138->144 \quad 0.49570$

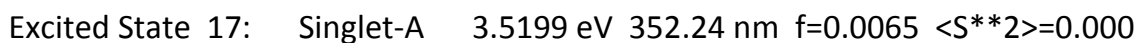

$134 \rightarrow 139 \quad 0.61027$

$138->142 \quad 0.15877$

$138->144 \quad 0.22717$

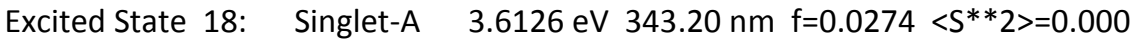

$134->140 \quad 0.66309$

$137->144 \quad 0.10075$

$138->143-0.10189$

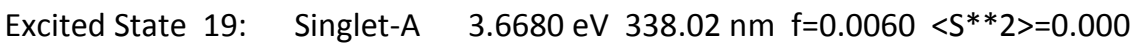
$133 \rightarrow 139 \quad 0.41947$

$133->140 \quad-0.27511$ 


$$
\begin{array}{rr}
135->140 & 0.14126 \\
135->143 & -0.13592 \\
137->143 & 0.41508 \\
138->144 & -0.13568
\end{array}
$$

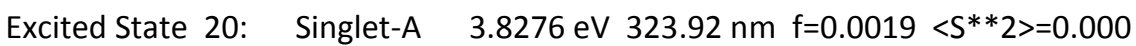
$132->139-0.17347$

$133 \rightarrow 141 \quad 0.12003$

$137->142 \quad 0.63615$

$\left[\left(\mathrm{P}(\mathrm{O}-i-\mathrm{Pr})_{3}\right)_{2} \mathrm{Rh}\left(\mathrm{MoS}_{4}\right)\right]^{-} \mathbf{8}$

\section{Excitation energies and oscillator strengths:}

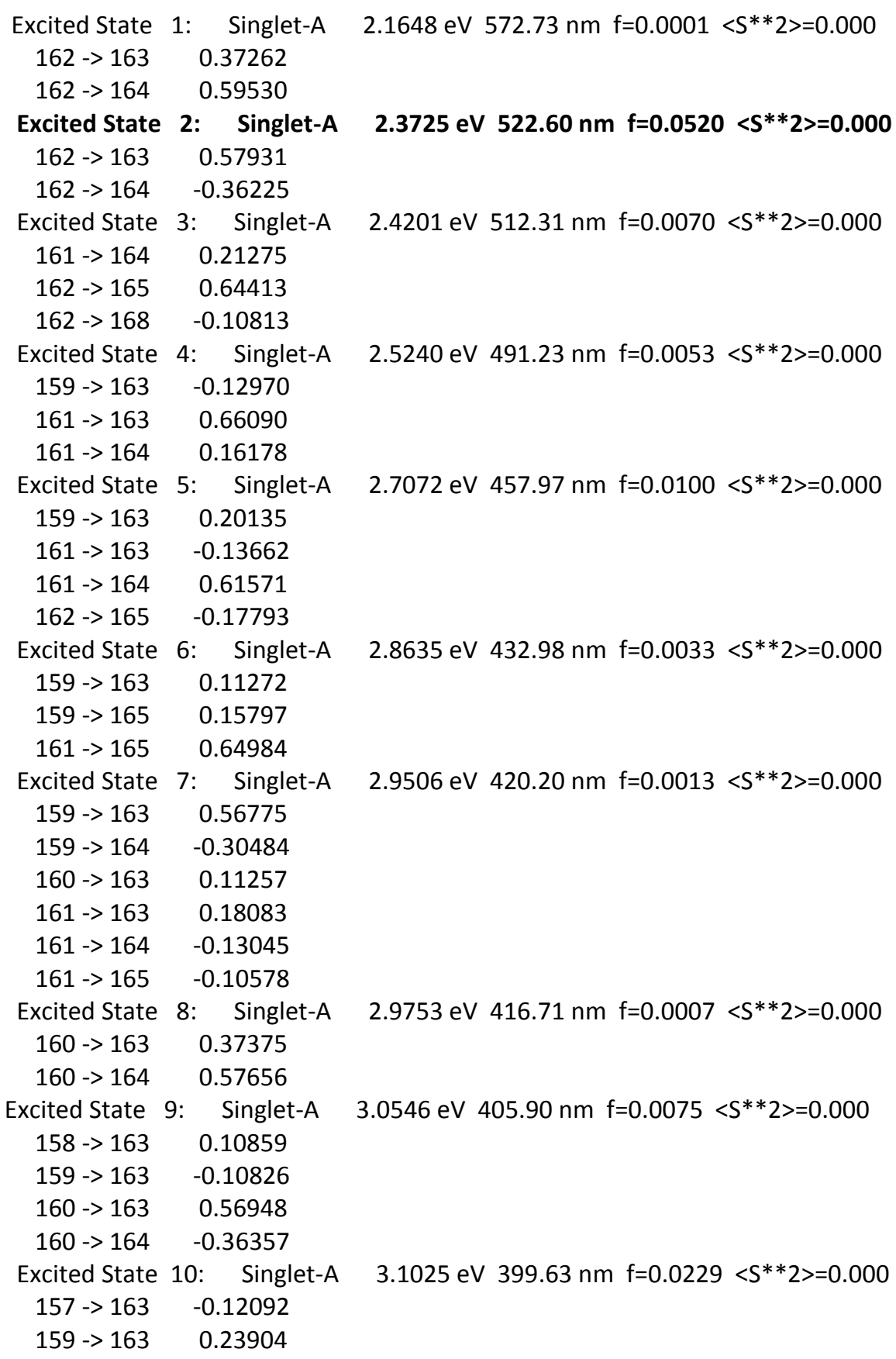




$$
\begin{array}{rr}
159->164 & 0.45913 \\
160->165 & 0.39028 \\
162->168 & -0.16354
\end{array}
$$

Excited State 11: Singlet-A $\quad 3.1679$ eV $391.38 \mathrm{~nm} \mathrm{f=0.0006<S**} 2>=0.000$

$$
\begin{array}{rr}
157->163 & 0.15052 \\
158->165 & 0.16908 \\
159->163 & -0.11528 \\
159 \rightarrow>164 & -0.35339 \\
160->165 & 0.49937 \\
160->168 & -0.12228
\end{array}
$$

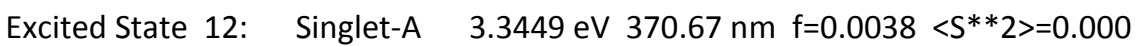
$\begin{array}{ll}160->164 & 0.10277 \\ 162->167 & 0.68344\end{array}$

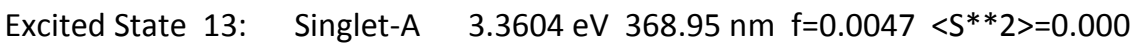
$158 \rightarrow 163 \quad 0.23078$

$162->166 \quad 0.64241$

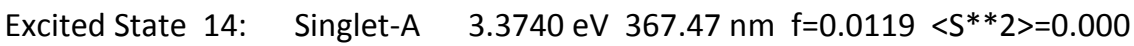
156 -> $163 \quad 0.11425$

$158 \rightarrow 163 \quad 0.62252$

$161 \rightarrow 166 \quad 0.10045$

$162->166-0.23570$

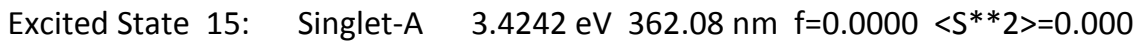
$158 \rightarrow 164 \quad-0.25031$

$159 \rightarrow 165 \quad 0.56391$

$161->165 \quad-0.17039$

$161 \rightarrow 168-0.19425$

$162->168-0.10917$

Excited State 16: Singlet-A 3.4535 eV $359.02 \mathrm{~nm} \mathrm{f}=0.0016<\mathrm{S}^{* *} 2>=0.000$ $157 \rightarrow 163-0.17075$

$158->164 \quad-0.19131$

$158 \rightarrow 165 \quad 0.16374$

$162 \rightarrow 165 \quad 0.11825$

$162->168 \quad 0.58944$

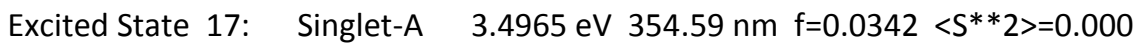
$158 \rightarrow 164 \quad 0.58968$

$159 \rightarrow 165 \quad 0.18761$

$161 \rightarrow 168 \quad-0.20023$

$162->168 \quad 0.13637$

Excited State 18: Singlet-A 3.5601 eV $348.26 \mathrm{~nm} \mathrm{f=0.0012}<\mathrm{S}^{* *} 2>=0.000$ $157 \rightarrow 163 \quad 0.36357$

$157 \rightarrow 164 \quad 0.54993$

$161->167 \quad 0.20439$

Excited State 19: $\quad$ Singlet-A $\quad 3.7089 \mathrm{eV} \quad 334.29 \mathrm{~nm} \mathrm{f}=0.0053<\mathrm{S}^{* *} 2>=0.000$ $157 \rightarrow 163 \quad 0.31821$

$157 \rightarrow 164-0.34128$

$158 \rightarrow 165-0.22093$

$159 \rightarrow 164 \quad 0.10547$

$159 \rightarrow 167 \quad-0.12155$

$161->167 \quad 0.39638$

$162->168 \quad 0.12274$

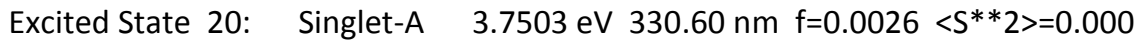

$156 \rightarrow 163-0.15361$

$156->165-0.14508$

$157 \rightarrow 164 \quad 0.13230$

$158 \rightarrow 165 \quad-0.25421$

$161->166 \quad 0.53364$

$161->167 \quad-0.16001$ 


\section{SI.6 QALE electronic parameters for $\mathbf{P}(\mathrm{O}-\mathrm{o}-\mathrm{Tol})_{3}$}

The QALE electronic parameters of the, not so common, tri(o-tolyl) phosphite are not listed in the literature. The estimation of the numerical value of $\chi_{\mathrm{d}}$ and $\pi_{\mathrm{p}}$ was achieved indirectly as follows.

\section{- Estimation of $\chi_{\mathrm{d}}$}

Numerical values of the $\chi_{\mathrm{d}}$ and the ${ }^{\mathrm{FT}} \mathrm{TEP}$ parameters for various phosphites are listed in the following Table.

$\begin{array}{lll}\text { Phosphite } & \chi_{\mathrm{d}}{ }^{a} & { }^{\mathrm{FT}} \mathrm{TEP}^{b} \\ \mathrm{P}(\mathrm{O}-i-\mathrm{Pr})_{3} & 13.4 & 19.05 \\ \mathrm{P}(\mathrm{OEt})_{3} & 15.8 & 21.60 \\ \mathrm{P}(\mathrm{OPr})_{3} & 15.9 & 20.90 \\ \mathrm{P}(\mathrm{OBu})_{3} & 15.9 & 20.85 \\ \mathrm{P}(\mathrm{OMe})_{3} & 17.9 & 24.10 \\ \mathrm{P}(\mathrm{OPh})_{3} & 23.6 & 30.20 \\ \mathrm{P}\left(\mathrm{O}-p-\mathrm{ClC}_{6} \mathrm{H}_{4}\right)_{3} & 27.2 & 33.10 \\ \mathrm{P}\left(\mathrm{O}-p-\mathrm{NCC}_{6} \mathrm{H}_{4}\right)_{3} & 31.7 & 37.65\end{array}$

${ }^{a}$ Fernandez, A. L.; Reyes, C.; Prock, A.; Giering, W. P. J. Chem. Soc., Perkin Trans. 2 2000, $1033-$ 1041.; ${ }^{b}$ Bartik, T.; Himmler, T.; Schulte, H.-G.; Seevogel, K. J. Organomet. Chem. 1984, 272, 29-41.

Since $\chi_{\mathrm{d}}$ correlates linearly with ${ }^{\mathrm{FT}} \mathrm{TEP}$ :

$\chi_{\mathrm{d}}=-4.60564+0.955628 \cdot{ }^{\mathrm{FT}} \mathrm{TEP}\left(\mathrm{R}^{2}=0.995\right)$

by assuming that the value of $\chi_{\mathrm{d}}$ for $\mathrm{P}(\mathrm{O}-o-\mathrm{Tol})_{3}$ can be estimated from equation SI.6.1, it follows that $\left({ }^{\mathrm{FT}} \mathrm{TEP}\left(\mathrm{P}(\mathrm{O}-o-\mathrm{Tol})_{3}\right)=29.05\right)$ :

for $\mathrm{P}(\mathrm{O}-o-\mathrm{Tol})_{3}: \chi_{\mathrm{d}}=-4.60564+0.955628 \cdot \mathbf{2 9 . 0 5} \Rightarrow \chi_{\mathrm{d}}=\mathbf{2 3 . 2}$

The variation of $\chi_{\mathrm{d}}$ for $\mathrm{P}(\mathrm{OPh})_{3}$ and $\mathrm{P}(\mathrm{O}-o-\mathrm{Tol})_{3}(23.6$ and 23.2) seems to be in accordance with the variation of $\chi_{\mathrm{d}}$ for the respective phosphines $\mathrm{PPh}_{3}$ and $\mathrm{P}(o-\mathrm{Tol})_{3}(13.25$ and 11.5).

- Estimation of $\pi_{\mathrm{p}}$

The examination of the correlation of $\Delta \delta(\mathrm{P})$ for complexes $4,6,7$, and 8 with respect to the QALE model electronic parameters $\chi_{\mathrm{d}}$ and $\pi_{\mathrm{p}}$ (Table 4 ), resulted in the following statistically significant correlation:

$\Delta \delta(\mathrm{P})=27.2296+1.15472 \cdot \chi_{\mathrm{d}}-13.9785 \cdot \pi_{\mathrm{p}}\left(\mathrm{R}^{2}=0.996\right)$

For $\mathrm{P}(\mathrm{O}-\mathrm{o}-\mathrm{Tol})_{3}: \mathbf{- 6 . 8 3}=27.2296+1.15472 \cdot \mathbf{2 3 . 2}-13.9785 \cdot \pi_{\mathrm{p}} \Rightarrow \pi_{\mathrm{p}}=\mathbf{4 . 3}$

- Estimation of $E_{\mathrm{ar}}$

The examination of the correlation of ${ }^{1} J$ for complexes $4,6,7$, and 8 with respect to the QALE model electronic parameters $\chi_{d}$ and $E_{\text {ar }}$ (Table 4), resulted in the following statistically significant correlation:

${ }^{1} J(\mathrm{Rh}-\mathrm{P})=181.166+2.4788 \cdot \chi_{\mathrm{d}}+32.4851 \cdot \pi_{\mathrm{p}}\left(\mathrm{R}^{2}=1.000\right)$

For $\mathrm{P}(\mathrm{O}-o-\mathrm{Tol})_{3}: \mathbf{2 8 3 . 2}=181.166+2.4788 \cdot 23.1+32.4851 \cdot \pi_{\mathrm{p}} \Rightarrow \boldsymbol{E}_{\mathrm{ar}}=\mathbf{1 . 4}$ 


\section{SI.7 Statistical Analysis of the QALE Multiple Regressions for complexes 4-8}

$$
\Delta \delta(\mathrm{P}) / p p m=(28.13 \pm 0.92)+(1.19 \pm 0.10) \cdot \chi_{\mathrm{d}}-(14.47 \pm 0.64) \cdot \pi_{\mathrm{p}}
$$

\begin{tabular}{|l|l|l|l|l|}
\hline Parameter & Estimate & $\begin{array}{l}\text { Standard } \\
\text { Error }\end{array}$ & $\begin{array}{l}T \\
\text { Statistic }\end{array}$ & P-Value \\
\hline CONSTANT & 28.1382 & 0.922385 & 30.5059 & 0.0011 \\
\hline$\chi_{\mathrm{d}}$ & 1.18668 & 0.104473 & 11.3588 & 0.0077 \\
\hline$\pi_{\mathrm{p}}$ & -14.4659 & 0.641214 & -22.5602 & 0.0020 \\
\hline
\end{tabular}

R-squared $=99.7996$ percent

$\Delta \delta(\mathrm{P}) / \mathrm{ppm} \cdot \Delta E / 10^{-4} \cdot \mathrm{cm}^{-1}=(52.27 \pm 1.76)+(2.21 \pm 0.20) \cdot \chi_{\mathrm{d}}-(26.98 \pm 1.22) \cdot \pi_{\mathrm{p}}$

\begin{tabular}{|l|l|l|l|l|}
\hline Parameter & Estimate & $\begin{array}{l}\text { Standard } \\
\text { Error }\end{array}$ & $\begin{array}{l}T \\
\text { Statistic }\end{array}$ & $P$-Value \\
\hline CONSTANT & 52.2651 & 1.75468 & 29.7861 & 0.0011 \\
\hline$\chi_{\mathrm{d}}$ & 2.21402 & 0.198741 & 11.1402 & 0.0080 \\
\hline$\pi_{\mathrm{p}}$ & -26.9752 & 1.2198 & -22.1145 & 0.0020 \\
\hline
\end{tabular}

R-squared $=99.7913$ percent

${ }^{1} J(\mathrm{Rh}-\mathrm{P}) / H z=(183.05 \pm 1.66)+(2.44 \pm 0.05) \cdot \chi_{\mathrm{d}}+(31.29 \pm 1.49) \cdot E_{\mathrm{ar}}$

\begin{tabular}{|l|l|l|l|l|}
\hline Parameter & Estimate & $\begin{array}{l}\text { Standard } \\
\text { Error }\end{array}$ & $\begin{array}{l}T \\
\text { Statistic }\end{array}$ & P-Value \\
\hline CONSTANT & 183.05 & 1.66077 & 110.22 & 0.0001 \\
\hline$\chi_{\mathrm{d}}$ & 2.44616 & 0.0543196 & 45.0327 & 0.0005 \\
\hline$E_{\mathrm{ar}}$ & 31.288 & 1.49098 & 20.9849 & 0.0023 \\
\hline
\end{tabular}

R-squared $=99.9511$ percent 